\title{
Recent developments and open problems in linear series
}

\author{
Th. Bauer, C. Bocci, S. Cooper, S. Di Rocco, M. Dumnicki, \\ B. Harbourne, K. Jabbusch, A. L. Knutsen, A. Küronya, R. Miranda, \\ J. Roé, H. Schenck, T. Szemberg, Z. Teitler
}

April 16, 2011

\section{Contents}

1 Introduction 2

2 Original problems

2.1 Asymptotic effectivity (B. Harbourne) . . . . . . . . . . . . 2

2.2 Semi-effectiveness (B. Harbourne) . . . . . . . . . . . . . . 3

2.3 Stability of speciality (T. Szemberg) . . . . . . . . . . . . 4

2.4 Regularity for generic monomial zero schemes (J. Roé) . . . . . . . . 4

2.5 Bounding cohomology (B. Harbourne) . . . . . . . . . . . . 6

2.6 Algebraic fundamental groups and Seshadri numbers (J.-M. Hwang) 6

2.7 Blow-ups of $\mathbb{P}^{n}$ and hyperplane arrangements (H. Schenck) . . . . 7

2.8 Bounds for symbolic powers $($ Z. Teitler $) \ldots \ldots \ldots$

3 Progress 9

3.1 Relating $h^{0}$ and $h^{1}$ on surfaces $\ldots \ldots \ldots \ldots$

3.2 Speciality on blow-ups of $\mathbb{P}^{2} \ldots \ldots \ldots \ldots \ldots$

3.3 Bounded negativity . . . . . . . . . . . . . . . . 13

3.4 Partial proof of Conjecture 3.3.5: Very Weak Bounded Negativity . . 15

3.5 Partial proof of Conjecture 3.3.4: Weak Bounded Negativity . . . . . 16

3.6 Bounded negativity conjecture and Seshadri constants . . . . . . . . 17

3.7 The Weighted Bounded Negativity Conjecture . . . . . . . . . . . . 19

3.8 Bounded negativity for reducible curves . . . . . . . . . . . . . 20

3.9 Imposing higher vanishing order at one point . . . . . . . . . . . 22

3.10 Geometrization of Dumnicki's method 12 . . . . . . . . . . 23

3.11 Linear systems connected to hyperplane arrangements . . . . . . . . 29

3.12 Limitations of multiplier ideal approach to bounds for symbolic powers 30

A Logarithmic differentials and the Miyaoka-Yau inequality 31

A.1 Basics . . . . . . . . . . . . . . . . . . . 31

A.2 The Miyaoka-Yau inequality for $\Omega_{X}^{1}(\log C) \ldots \ldots \ldots$ 


\section{Introduction}

In the week of October 3-9, 2010, the Mathematisches Forschungsinstitut at Oberwolfach hosted the Mini-Workshop "Linear Series on Algebraic Varieties." These notes contain a variety of interesting problems which motivated the participants prior to the event, and examples, results and further problems which grew out of discussions during and shortly after the workshop. Many arguments presented here are scattered in the literature or constitute "folklore." It was one of our aims to have a usable and easily accessible collection of examples and results 1

\section{Original problems}

We begin with a list of problems which were suggested by the participants for the Mini-Workshop. This list was discussed for three months before the workshop began.

\subsection{Asymptotic effectivity (B. Harbourne)}

Let $S=\left\{p_{1}, \ldots, p_{r}\right\}$ be distinct points in $\mathbb{P}^{N}$, over an algebraically closed ground field $k$ of arbitrary characteristic. Let $f: X \rightarrow \mathbb{P}^{N}$ be the morphism obtained by blowing up $p_{1}, \ldots, p_{r}$, and denote the exceptional divisors by $E_{1}, \ldots, E_{r}$. Let $H=f^{*}\left(\mathcal{O}_{\mathbb{P}^{N}}(1)\right)$ and let $L(d, m)=d H-m\left(E_{1}+\cdots+E_{r}\right)$. Waldschmidt 56 ] introduced and showed the existence of the following quantity:

$$
e(S):=\lim _{m \rightarrow \infty} \frac{a(S, m)}{m}
$$

where $a(S, m):=\min \left\{d: h^{0}(X, L(d, m))>0\right\}$. It follows from the proof that $m e(S) \leqslant a(S, m)$ for all $m \geqslant 1$.

Problem 2.1.1 Develop computational or conceptual methods for evaluating, estimating or bounding $e(S)$.

It is trivial to show that $a(S, m)=r m$ and hence $e(S)=r$ when $N=1$. It is an open problem in general to compute $e(S)$ when $N>1$. For example, for $r>9$ generic points $p_{i} \in \mathbb{P}^{2}$, a still open conjecture of Nagata [40, Conjecture, p. 772] is equivalent to having $e(S)=\sqrt{r}$.

Using complex analytic methods, Waldschmidt [56] and Skoda [47] showed that

$$
\frac{a(S, 1)}{N} \leqslant e(S)
$$

Given $k \geqslant 0$, by [28, Theorem 1.1(a)], for any finite subset $S \subset \mathbb{P}^{N}$ and all $m \geqslant 1$ we have

$$
\frac{a(S, k+1)}{k+N} \leqslant \frac{a(S, m(N+k))}{m(k+N)}
$$

\footnotetext{
${ }^{1}$ Cooper's participation was supported by the "US Junior Oberwolfach Fellows" joint NSF-MFO program under NSF grant DMS-0540019. Partial support during this project is kindly acknowledged as follows: Bocci by Italian PRIN funds; Di Rocco by Vetenskapsådets grant NT:2006-3539; Küronya by DFG-Forschergruppe 790 "Classification of Algebraic Surfaces and Compact Complex Manifolds" and the OTKA grants 77476 and 77604 by the Hungarian Academy of Sciences; Roé by Spanish Ministerio de Educación y Ciencia, grant MTM2009-10359; Schenck by NSF 07-07667, NSA 90403-1-0006; Szemberg by MNiSW grant N N201 388834
} 
and hence

$$
\frac{a(S, k+1)}{k+N} \leqslant e(S) \leqslant \frac{a(S, k+1)}{k+1} .
$$

Taking $k=0$ recovers the Waldschmidt-Skoda bound (1). Taking large values of $k$ gives one a way of computing arbitrarily accurate estimates of $e(S)$ [24], but computing $a(S, k)$ for large $k$ is difficult to do.

Chudnovsky [8] conjectured (and proved for $N=2$ ) the stronger bound

\section{Conjecture 2.1.2}

$$
\frac{a(S, 1)+N-1}{N} \leqslant e(S) .
$$

(For the proof when $N=2$, reduce to the case that $r=\left(\begin{array}{c}a+1 \\ 2\end{array}\right)$, where $a=a(S, 1)$, and use the fact that then $L(a, 1)$ is nef.)

Two examples are known where equality in (2.1.2) holds: when the points lie in a hyperplane, or when $S$ is a star configuration [4] (i.e., given a set of $s$ hyperplanes in $\mathbb{P}^{N}$ such that at most $N$ of these hyperplanes meet at any single point, $S$ is the set of $r=\left(\begin{array}{l}s \\ N\end{array}\right)$ points at which $N$ of the hyperplanes meet).

Problem 2.1.3 If for some $m$ we have

$$
\frac{a(S, m)}{m}=\frac{a(S, 1)+N-1}{N}
$$

is it true that $S$ is either contained in a hyperplane or is a star configuration? What if

$$
e(S)=\frac{a(S, 1)+N-1}{N} ?
$$

Bocci and Chiantini [3] show for $N=2$ that $\frac{a(S, 2)}{2}=\frac{a(S, 1)+1}{2}$ implies that $S$ is either a set of collinear points or a star configuration.

\subsection{Semi-effectiveness (B. Harbourne)}

Again let the ground field $k$ be an algebraically closed field of arbitrary characteristic.

Definition 2.2.1 Let $X$ be an algebraic variety and $L$ a line bundle on $X$. We say that $L$ is semi-effective, if there exists $n>0$ such that $h^{0}(n L)>0$.

Let $p_{1}, \ldots, p_{r}$ be distinct points in $\mathbb{P}^{N}$. Let $f: X \rightarrow \mathbb{P}^{N}$ be obtained by blowing up $p_{1}, \ldots, p_{r}$ with the exceptional divisors being $E_{1}, \ldots, E_{r}$. Let

$$
D=d H-m_{1} E_{1}-\cdots-m_{r} E_{r}
$$

where $H$ is the pullback via $f$ of a general hyperplane.

The following question was raised by M. Velasco and D. Eisenbud (in an email from Velasco to Harbourne, November 2009).

Problem 2.2.2 Is there a way to determine if $D$ is semi-effective?

For a specific problem consider

$$
D=13 L-5 E_{1}-4 E_{2}-\cdots-4 E_{10}
$$


for generic points $p_{1}, \ldots, p_{10} \in \mathbb{P}^{2}$. M. Dumnicki and J. Roé have independently shown that this system is not semi-effective (see Section 3.10). But there are infinitely many more similar examples for which semi-effectivity is still not known. For example, given generic points $p_{i} \in \mathbb{P}^{2}$, consider $D=111 L-36 E_{1}-35 E_{2}-\cdots-35 E_{10}$ or more generally $D=d L-(m+1) E_{1}-m E_{2}-\cdots-m E_{10}$, where $d=\left(b^{2}+a^{2}\right) / 2$, $m+1=b a, m=\left(b^{2}-a^{2}\right) / 6$ and where $0<a<b$ are odd integers satisfying $(a+3 b)^{2}-10 b^{2}=6$. Note that $D^{2}=0$ but $D$ cannot be reduced by Cremona transformations. According to the SHGH Conjecture [46, 23, 20, 27], we expect that none of these $D$ are semi-effective.

Limits like those of Waldschmidt are relevant to Problem 2.2.2, Let $a\left(\sum_{i=1}^{r} m_{i} p_{i}\right)$ be the least $t$ such that $h^{0}\left(t L-\sum_{i} m_{i} E_{i}\right)>0$ and define

$$
e\left(\sum_{i} m_{i} p_{i}\right):=r \lim _{t} \frac{a\left(t \sum_{i} m_{i} p_{i}\right)}{t \sum_{i} m_{i}} .
$$

Then $D=d L-\sum_{i=1}^{r} m_{i}$ is semi-effective if $e\left(\sum_{i} m_{i} p_{i}\right) / r<d / \sum_{i} m_{i}$, and it is not semi-effective if $e\left(\sum_{i} m_{i} p_{i}\right) / r>d / \sum_{i} m_{i}$. It is not clear whether $D$ is semi-effective when $e\left(\sum_{i} m_{i} p_{i}\right) / r=d / \sum_{i} m_{i}$. But, if the SHGH Conjecture is true, this boundary case is precisely the situation of the examples in the preceding paragraph.

\subsection{Stability of speciality (T. Szemberg)}

Let $p_{1}, \ldots, p_{r}$ be points in the projective plane. Let $f: X \rightarrow \mathbb{P}^{2}$ be the blow-up of $p_{1}, \ldots, p_{r}$ with exceptional divisors $E_{1}, \ldots, E_{r}$. Let $H=f^{*}\left(\mathcal{O}_{\mathbb{P}^{2}}(1)\right)$, and let

$$
D=d H-m_{1} E_{1}-\cdots-m_{r} E_{r} .
$$

Assume that the divisor $D$ is special (i.e., $D$ is effective with $h^{1}(X, D)>0$ ). Is it then true that

$$
n D \text { is special for all } n>1 \text { ? }
$$

A somewhat more demanding problem is to determine whether the asymptotic cohomology function $\widehat{h}^{1}$ as defined in [33] is positive. This is not true if the points are arbitrary (or less than 10?). Indeed, let $p_{1}, \ldots, p_{9}$ be intersection points of two cubics and let $L=3 H-\sum_{i=1}^{9} E_{i}$ be the anti-canonical pencil. Then

$$
h^{0}(n L)=n+1 \text { and } h^{1}(n L)=n \text { and } h^{2}(n L)=0,
$$

so that all $n L$ are special but all asymptotic cohomology functions vanish.

\subsection{Regularity for generic monomial zero schemes (J. Roé)}

Let $I \subset k[x, y]$ be a monomial ideal, and let $Z=\operatorname{Spec} k[x, y] / I$. Let $n=\operatorname{dim}_{k} k[x, y] / I$ be the length of $Z$, which we assume to be finite (in this case, $I$ is $(x, y)$-primary, and $Z$ is supported at the origin). For each (irreducible smooth projective) surface $S$ defined over $k$ there is an irreducible constructible subset $\operatorname{Hilb}_{I} S$ of $\operatorname{Hilb}^{n} S$ whose closed points are the subschemes $Y$ of $S$ isomorphic to $Z$.

Of course, if $I=(x, y)^{m}$ then $Z$ is just an $m$-fold point, and $\operatorname{Hilb}_{I} S \cong S$ is the set of points of $S$ taken with multiplicity $m$. If $I=\left(y^{2}, y x^{2}, x^{3}\right)$ then $Z$ is a cusp scheme, which means that curves containing $Z$ have at least a cusp at the point supporting $Z$ (i.e., they have a cusp or a more special singularity, and generically it 
is a cusp). Since the cusp scheme marks the tangent direction to the cusp, $\operatorname{Hilb}_{I} S$ is in this case naturally isomorphic to the (projectivized) tangent bundle of $S$. Other monomial ideals correspond to other singularity "types."

Problem 2.4.1 Describe $\operatorname{Hilb}_{I} S$. Is it locally closed? What adjacencies are there between such subsets of the Hilbert scheme?

$\mathrm{As} \mathrm{Hilb}_{I} S$ is irreducible, it makes sense to consider general (or very general, or generic) subschemes $Y$ of $S$ isomorphic to $Z$. For each divisor $D$ we have an exact sequence

$$
0 \rightarrow \mathcal{I}_{Y} \otimes \mathcal{O}_{S}(D) \rightarrow \mathcal{O}_{S}(D) \rightarrow \mathcal{O}_{Y}(D) \rightarrow 0
$$

inducing the usual exact sequence in cohomology.

Problem 2.4.2 What can we say (or conjecture) about the cohomology of $\mathcal{I}_{Y} \otimes$ $\mathcal{O}_{S}(D)$ ? To be more precise, it would be nice to have conditions on $S, I$ and $D$ implying that $h^{0}=\max \left\{0, h^{0}\left(\mathcal{O}_{S}(D)\right)-n\right\}$, in which case $h^{1}$ and $h^{2}$ are easily computed by Riemann-Roch and duality.

Assuming the previous problem is understood, one can then further ask about the base locus of global sections of $\mathcal{I}_{Y} \otimes \mathcal{O}_{S}(D)$, and ask if they cut out the scheme $Z$. Such questions are of interest in the construction of curves with imposed singularities, and have been studied mainly for schemes of small multiplicity (the multiplicity of $Z$ is the maximum $m$ such that $\left.I \subset(x, y)^{m}\right)$. Note that multiplicity 1 schemes are curvilinear and well known; multiplicity 2 monomial schemes are also quite well understood [43. The same kind of questions arise as auxiliary problems for the induction arguments of differential Horace methods [15], even when one is primarily interested in linear series defined by ordinary multiple points.

Note also that the scheme defined by $I=\left(y, x^{r}\right)^{m}$ is a specialization (or collision) of $r$ distinct $m$-fold points; thus the dimension of the linear series $H^{0}\left(\mathcal{I}_{Y} \otimes \mathcal{O}_{S}(D)\right)$ is by semicontinuity a bound for the dimension of the linear series determined by a set of $r$ general $m$-fold points. This gives a link with the Nagata conjecture [40] and the SHGH conjecture [46, 23, 20, 27]. More precisely:

Conjecture 2.4.3 Let $S=\mathbb{P}^{2}$ and $I=\left(y, x^{r}\right)^{m}$, where $r$ and $m$ are natural numbers with $r>9$. Then for all $d>0$, and general $Y \in \operatorname{Hilb}_{I} S$,

$$
H^{0}\left(\mathcal{I}_{Y} \otimes \mathcal{O}_{\mathbb{P}^{2}}(D)\right)=\max \left\{0,\left(\begin{array}{c}
d+2 \\
2
\end{array}\right)-r\left(\begin{array}{c}
m+1 \\
2
\end{array}\right)\right\}
$$

Conjecture 2.4.4 Let $S=\mathbb{P}^{2}$ and $I=\left(y, x^{r}\right)^{m}$, where $r$ and $m$ are natural numbers with $r>9$. Then for all $d \leqslant m \sqrt{r}$, and general $Y \in \operatorname{Hilb}_{I} S, H^{0}\left(\mathcal{I}_{Y} \otimes \mathcal{O}_{\mathbb{P}^{2}}(D)\right)=$ 0 .

Conjecture 2.4.3 implies the uniform Harbourne-Hirschowitz conjecture, and Conjecture 2.4.4 implies Nagata's conjecture. It is also clear that Conjecture 2.4.3 implies Conjecture 2.4.4.

A conjecture in terms of monomial ideals implying the general HarbourneHirschowitz conjecture (without uniformity assumptions on the multiplicities) can be stated similarly; we skip it here to avoid introducing the necessary notations, which would lengthen this section unnecessarily, and refer to Hirschowitz's description of the "collisions de front" in [26] instead. 


\subsection{Bounding cohomology (B. Harbourne)}

There are various equivalent versions of the SHGH Conjecture [46, 23, 20, 27]. Here's one:

Conjecture 2.5.1 (SHGH) Let $C \subset X$ be a prime divisor where $X \rightarrow \mathbb{P}^{2}$ is the blow-up of generic points $p_{1}, \ldots, p_{s}$. Then $h^{1}\left(X, \mathcal{O}_{X}(C)\right)=0$.

Problem 2.5.2 How can we remove the assumption about the points being generic in the SHGH Conjecture?

The following conjecture arose out of discussions between Harbourne, J. Roé, C. Ciliberto and R. Miranda. [NB: Corollary 3.1 .2 gives a counterexample. See also Proposition 3.1.3.]

Conjecture 2.5.3 Let $X$ be a smooth projective surface (either rational or assume the characteristic is 0$)$. Then there exists a constant $c_{X}$ such that for every prime divisor $C$ we have $h^{1}(X, C) \leqslant c_{X} h^{0}(X, C)$.

The SHGH Conjecture is that $c_{X}=0$ when $X$ is obtained by blowing up generic points of $\mathbb{P}^{2}$.

\subsection{Algebraic fundamental groups and Seshadri numbers (J.-M. Hwang)}

Denote by $\widehat{\pi}_{1}(Y)$ the algebraic fundamental group of an irreducible variety $Y$. Following [32, Definition (2.7.1)], we say that a projective manifold $X$ has large algebraic fundamental group if for every irreducible variety $Z \subset X$ and its normalization $\nu: \bar{Z} \rightarrow X$, the image of the induced homomorphism on the algebraic fundamental groups

$$
\nu_{*}: \widehat{\pi}_{1}(\bar{Z}) \rightarrow \widehat{\pi}_{1}(X)
$$

is infinite. This is equivalent to saying that the algebraic universal cover of $X$ does not contain a complete subvariety. The proof of [32, Lemma 8.2] gives the following.

Proposition 2.6.1 Let $N$ be a positive number. Let $X$ be a projective manifold with large algebraic fundamental group and let $L$ be an ample line bundle on $X$. Then there exists a finite étale cover $p: X^{\prime} \rightarrow X$ such that any irreducible subvariety $W$ in $X^{\prime}$ satisfies $\left(p^{*} L\right)^{\operatorname{dim}(W)} \cdot W>N$.

One can then ask the following:

Problem 2.6.2 Let $X$ be a projective manifold with large algebraic fundamental group and let $L$ be an ample line bundle on $X$.

(1) Given a positive number $N$, does there exist a finite étale cover $p: X^{\prime} \rightarrow X$ such that the Seshadri number of $p^{*} L$ at any point is bigger than $N$ ?

(2) Given a positive number $N$, does there exist a finite étale cover $p: X^{\prime} \rightarrow X$ such that denoting by $\widetilde{p}: X^{\prime} \times X^{\prime} \rightarrow X \times X$ the self-product of $p$ and $D^{\prime} \subset$ $X^{\prime} \times X^{\prime}$ the diagonal, the Seshadri number of $\widetilde{p}^{*}\left(p_{1}^{*} L \otimes p_{2}^{*} L\right)$ along $D^{\prime}$ is bigger than $N$ ?

(3) If the answer to (1) or (2) is negative or unclear, what is the condition on the fundamental group of $X$ to guarantee a positive answer? 


\subsection{Blow-ups of $\mathbb{P}^{n}$ and hyperplane arrangements (H. Schenck)}

Let

$$
\mathcal{A}=\bigcup_{i=1}^{d} V\left(\alpha_{i}\right) \subseteq \mathbb{P}^{2}
$$

be a union of lines in $\mathbb{P}^{2}, Y$ the singular locus, and $\pi: X \rightarrow \mathbb{P}^{2}$ the blow-up at $Y$. Let $R=\mathbb{C}\left[y_{1}, \ldots, y_{d}\right]$, and for each linear dependency $\Lambda=\sum_{j=1}^{k} c_{i_{j}} \alpha_{i_{j}}=0$ on the lines of $\mathcal{A}$, let

$$
f_{\Lambda}=\sum_{j=1}^{k} c_{i_{j}}\left(y_{i_{1}} \cdots \widehat{y}_{i_{j}} \cdots y_{i_{k}}\right) .
$$

The ideal $I$ generated by the $f_{\Lambda}$ is called the Orlik-Terao ideal, and the quotient $C(\mathcal{A})=R / I$ is called the Orlik-Terao algebra; of course, $C(\mathcal{A})$ can be defined for arrangements in higher dimensional spaces. For a real, affine arrangement $\mathcal{A}$, Aomoto conjectured a relationship between $C(\mathcal{A})$ and the topology of $\mathbb{R}^{n} \backslash \mathcal{A}$, which Orlik and Terao proved in [42].

Example 2.7.1 Consider $\mathcal{A}=V\left(x_{1} x_{2} x_{3}\left(x_{1}+x_{2}+x_{3}\right)\left(x_{1}+2 x_{2}+3 x_{3}\right)\right) \subseteq \mathbb{P}^{2}$. Since $\alpha_{1}+\alpha_{2}+\alpha_{3}-\alpha_{4}=0$,

$$
y_{2} y_{3} y_{4}+y_{1} y_{3} y_{4}+y_{1} y_{2} y_{4}-y_{1} y_{2} y_{3} \in I .
$$

The five lines meet in ten points, and every subset of four lines gives a similar relation, one of which is redundant. Thus, $I$ is generated by four cubics, which turn out to be the maximal minors of a matrix of linear forms. This means that $I$ has a Hilbert-Burch resolution, and $V(I)$ is a surface of degree six in $\mathbb{P}^{4}$; a computation shows $V(I)$ has five singular points. Consider the divisor

$$
D_{\mathcal{A}}=4 E_{0}-\sum_{i=1}^{10} E_{i}
$$

on $X$, where $X$ is the blow-up of $\mathbb{P}^{2}$ at the ten points of $Y, E_{i}$ are the exceptional curves over the singular points, and $E_{0}$ is the proper transform of a line. Then $D_{\mathcal{A}}$ is nef but not ample; the lines of the original arrangement are contracted to points, and $I$ is the ideal of $X$ in $\operatorname{Proj}\left(H^{0}\left(D_{\mathcal{A}}\right)\right)$.

The example above is representative of the general case. In [45], it is shown that if

$$
\varphi_{\mathcal{A}}: X \rightarrow \mathbb{P}\left(H^{0}\left(D_{\mathcal{A}}\right)^{\vee}\right),
$$

then $C(\mathcal{A})$ is the homogeneous coordinate ring of $\varphi_{\mathcal{A}}(X)$ and $\varphi_{\mathcal{A}}$ is an isomorphism on $\pi^{*}\left(\mathbb{P}^{2} \backslash \mathcal{A}\right)$, contracts the lines of $\mathcal{A}$ to points, and blows up $Y$.

The motivation for studying $C(\mathcal{A})$ arises from its connection to topology. In [41, Orlik and Solomon determined the cohomology ring of a complex, affine arrangement complement $M=\mathbb{C}^{n+1} \backslash \mathcal{A}: A=H^{*}(M, \mathbb{Z})$ is the quotient of the exterior algebra $E=\bigwedge\left(\mathbb{Z}^{d}\right)$ on generators $e_{1}, \ldots, e_{d}$ in degree 1 by the ideal generated by all elements of the form $\partial e_{i_{1} \ldots i_{r}}:=\sum_{q}(-1)^{q-1} e_{i_{1}} \cdots \widehat{i_{i_{q}}} \cdots e_{i_{r}}$, for which codim $H_{i_{1}} \cap \cdots \cap H_{i_{r}}<r$. Since $A$ is a quotient of an exterior algebra, multiplication by an element $a \in A^{1}$ gives a degree one differential on $A$, yielding a cochain complex $(A, a)$ :

$$
(A, a): \quad 0 \longrightarrow A^{0} \stackrel{a}{\longrightarrow} A^{1} \stackrel{a}{\longrightarrow} A^{2} \stackrel{a}{\longrightarrow} \cdots \stackrel{a}{\longrightarrow} A^{\ell} \longrightarrow 0
$$


The first resonance variety $R^{1}(\mathcal{A})$ consists of points $a=\sum_{i=1}^{d} a_{i} e_{i} \leftrightarrow\left(a_{1}: \cdots: a_{d}\right)$ in $\mathbb{P}\left(A^{1}\right) \cong \mathbb{P}^{d-1}$ for which $H^{1}(A, a) \neq 0$. Conjectures of Suciu [49] relate the fundamental group of $M$ to $R^{1}(\mathcal{A})$. Falk showed that $R^{1}(\mathcal{A})$ may be described in terms of combinatorics, and conjectured that $R^{1}(\mathcal{A})$ is a subspace arrangement, which was shown by Cohen-Suciu ([9]) and Libgober-Yuzvinsky ([36]). The paper [45] describes a connection between combinatorics of $R^{1}(\mathcal{A})$ which give rise to factorizations of $D_{\mathcal{A}}$ and corresponding determinantal equations in $I$.

Problem 2.7.2 Do the results for lines in $\mathbb{P}^{2}$ generalize to higher dimension? For example, if $\mathcal{A} \subseteq \mathbb{P}^{n}$, then [45] shows that the Castelnuovo-Mumford regularity of $C(\mathcal{A})$ is bounded by $n$. Can an explicit description of the graded betti numbers of $C(\mathcal{A})$ be given in terms of the geometry of $\mathcal{A}$ ?

\subsection{Bounds for symbolic powers (Z. Teitler)}

Let $X$ be a non-singular variety of dimension $n$ defined over the complex numbers and let $Z \subseteq X$ be a reduced subscheme of $X$ with ideal sheaf $I=I_{Z} \subseteq \mathcal{O}_{X}$. The $p^{\text {th }}$ symbolic power of $I$, denoted $I^{(p)}$, is the sheaf of all function germs vanishing to order $\geqslant p$ at each point of $Z$. The inclusion $I^{p} \subseteq I^{(p)}$ is clear, or in other words $I^{r} \subseteq I^{(m)}$ for $r \geqslant m$, but it is not clear when inclusion in the other direction, $I^{(m)} \subseteq I^{r}$, holds; $m \geqslant r$ is necessary but not sufficient in general. Related to a result of Swanson [50], Ein-Lazarsfeld-Smith used multiplier ideals to prove that if every component of $Z$ has codimension $\leqslant e$ in $X$ then $I^{(r e)} \subseteq I^{r}$ for all $r \in \mathbb{N}$; in particular $I^{(r n)} \subseteq I^{r}[13$. This was subsequently proved in greater generality by Hochster-Huneke using the theory of tight closure [28].

The big height of a radical ideal $I$, denoted bight $(I)$, is the maximum codimension of a component of $V(I)$. Harbourne raised the question whether it is possible to give an improvement of the form $I^{(m)} \subseteq I^{r}$ whenever $m \geqslant f(r)$, for some function $f(r) \leqslant r e$, for all radical ideals of big height $e$. Bocci-Harbourne [4] showed if $\gamma>0$ is such that for all radical ideals $I, I^{(m)} \subseteq I^{r}$ whenever $m \geqslant \gamma r$, then $\gamma \geqslant n$. Furthermore, if $I^{(m)} \subseteq I^{r}$ holds whenever $m \geqslant \gamma r$ for all radical ideals $I$ with $\operatorname{bight}(I)=e$, then $\gamma \geqslant e$. This shows that the function $f(r)=r e$ appearing in the Ein-Lazarsfeld-Smith result cannot be decreased by lowering the coefficient $e=\operatorname{bight}(I)$.

Harbourne asked whether a constant term can be subtracted, that is whether $I^{(m)} \subseteq I^{r}$ holds whenever $m \geqslant f(r)=e r-k$ for all radical ideals of big height $e$, for some $k$. Values $k \geqslant e$ do not work (the containment fails if $I$ is a complete intersection and - rather trivially - if $r=1$ or $e=1)$. On the other hand, examples studied by Bocci-Harbourne suggest that $k=e-1$ might work. Harbourne made the following:

Conjecture 2.8.1 ([2, Conjecture 8.4.3]) For radical ideals I of big height e, $I^{(m)} \subseteq I^{r}$ whenever $m \geqslant$ re $-(e-1)$.

A weaker result was observed by Takagi-Yoshida [52] (see also [53] for an expository account) who showed that if $\ell<\operatorname{lct}\left(I^{(\bullet)}\right)$ then $I^{(m)} \subseteq I^{r}$ whenever $m \geqslant r e-\ell$. Here $\operatorname{lct}\left(I^{\bullet}\right)$ is the $\log$ canonical threshold of the graded system of ideals $I^{(\bullet)}$; in particular this is always $\leqslant e($ so $\ell \leqslant e-1)$.

Harbourne-Huneke have asked if an improvement is possible on the other side of the inclusion: Instead of asking for $I^{(m)} \subseteq I^{r}$, they raise the following: 
Problem 2.8.2 Suppose $(R, \mathfrak{m})$ is a regular local ring of dimension $n$ and $I \subseteq R$ is an ideal with $\operatorname{bight}(I)=e$. Then do the following hold?

1. $I^{(m)} \subseteq \mathfrak{m}^{r n-r} I^{r}$ for $m \geqslant r n$.

2. $I^{(m)} \subseteq \mathfrak{m}^{r n-r-(n-1)} I^{r}$ for $m \geqslant r n-(n-1)$.

3. $I^{(m)} \subseteq \mathfrak{m}^{r e-r} I^{r}$ for $m \geqslant r e$.

4. $I^{(m)} \subseteq \mathfrak{m}^{r e-r-(e-1)} I^{r}$ for $m \geqslant r e-(e-1)$.

\section{Progress}

In this part we show several solutions to the original problems, present examples which are closely related to the problems, and provide some evidence either for positive or negative answers or forcing reformulation of the original statements.

\subsection{Relating $h^{0}$ and $h^{1}$ on surfaces}

We show that Conjecture 2.5.3 is false in general. We claim (see Corollary 3.1.2) that there exists a surface $X$ such that for an arbitrary positive integer $c$ there exists a reduced, irreducible curve $C \subset X$ with

$$
h^{1}\left(X, \mathcal{O}_{X}(C)\right)>c \cdot h^{0}\left(X, \mathcal{O}_{X}(C)\right) .
$$

It turns out that an example of Kollár (taken from [35, Example 1.5.7]) provides a counterexample to Conjecture 2.5.3. We recall briefly the construction, in which we will closely follow the exposition mentioned above. We consider an elliptic curve $E$ without complex multiplication, and take the abelian surface

$$
Y:=E \times E
$$

to be our starting point. Divisors and the various cones on $Y$ are well understood. The Picard number $\rho(Y)$ equals 3 , and $N^{1}(X)_{\mathbb{R}}$ has the fairly natural basis consisting of the classes of $F_{1}, F_{2}$ (the fibres of the two projection morphisms), and that of the diagonal $\Delta \subseteq E \times E$. The intersection form on $Y$ is given by the numbers

$$
\left(F_{1}^{2}\right)=\left(F_{2}^{2}\right)=\left(\Delta^{2}\right)=0,\left(F_{1} \cdot F_{2}\right)=\left(F_{1} \cdot \Delta\right)=\left(F_{2} \cdot \Delta\right)=1 .
$$

It is known (for details see for example [35, Section 1.5.B]) that the nef and pseudoeffective cones coincide, and a class $C=a_{1} F_{1}+a_{2} F_{2}+b \Delta$ is nef if and only if $\left(C^{2}\right) \geqslant 0$ and $(C \cdot H) \geqslant 0$ for some ample class $H$. In coordinates we can express this as

$$
a_{1} a_{2}+a_{1} b+a_{2} b \geqslant 0 \text { and } a_{1}+a_{2}+b \geqslant 0
$$

by choosing the ample divisor $F_{1}+F_{2}+\Delta$ for $H$.

For every integer $n \geqslant 2$ set

$$
A_{n}:=n F_{1}+\left(n^{2}-n+1\right) F_{2}-(n-1) \Delta .
$$

It is immediate to check that

1. $\left(A_{n}^{2}\right)=2$, and 
2. $\left(A_{n} \cdot\left(F_{1}+F_{2}\right)\right)=n^{2}-2 n+3>0$.

Kollár now sets $R:=F_{1}+F_{2}$, and picks a smooth divisor $B \in|2 R|$ (which exists because $2 R$ is base point free by the Lefschetz theorem [34, Theorem 4.5.1]) to form the double cover $f: X \rightarrow Y$ branched along $B$. Let $D_{n}:=f^{*} A_{n}$.

Proposition 3.1.1 With notation as above,

$$
h^{1}\left(X, \mathcal{O}_{X}\left(n D_{n}\right)\right) \geqslant n^{3}-2 n^{2}+3 n-1 .
$$

Proof. We will estimate $h^{1}\left(X, \mathcal{O}_{X}\left(n D_{n}\right)\right)$ from below with the help of the Leray spectral sequence. It is a standard fact that

$$
E_{2}^{p, q}:=H^{p}\left(Y, R^{q} f_{*} \mathcal{O}_{X}\left(n D_{n}\right)\right) \Longrightarrow H^{p+q}\left(X, \mathcal{O}_{X}\left(n D_{n}\right)\right) .
$$

We are interested in the case $p+q=1$, in which case the involved terms are

$$
H^{0}\left(Y, R^{1} f_{*}\left(\mathcal{O}_{X}\left(n D_{n}\right)\right) \text { and } H^{1}\left(Y, f_{*} \mathcal{O}_{X}\left(n D_{n}\right)\right)\right. \text {. }
$$

Of these the second term survives unchanged to the $E_{\infty}$ term, and so we obtain an inclusion

$$
H^{1}\left(Y, f_{*} \mathcal{O}_{X}\left(n D_{n}\right)\right) \hookrightarrow H^{1}\left(X, \mathcal{O}_{X}\left(n D_{n}\right)\right) .
$$

It is $H^{1}\left(Y, f_{*} \mathcal{O}_{X}\left(n D_{n}\right)\right)$ that we will estimate from below. By [35, Proposition 4.1.6] on the properties of cyclic coverings, one has

$$
f_{*} \mathcal{O}_{X}=\mathcal{O}_{Y} \oplus \mathcal{O}_{Y}(-R)
$$

which implies

$$
f_{*}\left(\mathcal{O}_{X}\left(n D_{n}\right)\right)=\mathcal{O}_{Y}\left(n A_{n}\right) \oplus \mathcal{O}_{Y}\left(n A_{n}-R\right)
$$

via the projection formula.

It follows that

$$
H^{1}\left(Y, f_{*}\left(\mathcal{O}_{X}\left(n D_{n}\right)\right)\right)=H^{1}\left(Y, \mathcal{O}_{Y}\left(n A_{n}\right)\right) \oplus H^{1}\left(Y, \mathcal{O}_{Y}\left(n A_{n}-R\right)\right) .
$$

We can determine the second term of the sum from the Riemann-Roch theorem. On the abelian surface $Y=E \times E$ one has $\chi\left(\mathcal{O}_{Y}\right)=0$ and $K_{Y}=\mathcal{O}_{Y}$, hence Riemann-Roch has the particularly simple form

$$
\chi\left(\mathcal{O}_{Y}\left(n A_{n}-R\right)\right)=\frac{1}{2}\left(n A_{n}-R\right)^{2} .
$$

We compute

$$
\begin{aligned}
\left(n A_{n}-R\right)^{2} & =n^{2} A_{n}^{2}-2 n\left(A_{n} \cdot R\right)+R^{2} \\
& =2 n^{2}-2 n\left(n^{2}-2 n+3\right)+2 \\
& =-2 n^{3}+6 n^{2}-6 n+2 \\
& =-2(n-1)^{3} \\
& <0,
\end{aligned}
$$

therefore neither $n A_{n}-R$ nor its negative is effective, resulting in

$$
H^{0}\left(Y, \mathcal{O}_{Y}\left(n A_{n}-R\right)\right)=0
$$

and

$$
H^{2}\left(Y, \mathcal{O}_{Y}\left(n A_{n}-R\right)\right)=H^{0}\left(Y, \mathcal{O}_{Y}\left(R-n A_{n}\right)\right)=0 .
$$

Hence we can conclude that

$$
h^{1}\left(X, \mathcal{O}_{X}\left(n D_{n}\right)\right) \geqslant h^{1}\left(Y, \mathcal{O}_{Y}\left(n A_{n}-R\right)\right)=n^{3}-2 n^{2}+3 n-1>0 .
$$


Corollary 3.1.2 With notation as above, for an arbitrary positive integer c there exists a prime divisor $C$ on $X$ such that

$$
h^{1}\left(X, \mathcal{O}_{X}(C)\right)>c \cdot h^{0}\left(X, \mathcal{O}_{X}(C)\right) .
$$

Proof. This is in fact a corollary of the proof of the Proposition 3.1.1. For $n \geqslant 2$ the linear system $\left|n A_{n}\right|$ is globally generated by the Lefschetz theorem [34, Theorem 4.5.1] and it is not composed with a pencil. The same holds true for $\left|n D_{n}\right|$, therefore the base-point free Bertini theorem implies that the general element of $\left|n D_{n}\right|$ is reduced and irreducible. Let $C_{n}$ be such an element. Then

$$
h^{0}\left(X, \mathcal{O}_{X}\left(C_{n}\right)\right)=h^{0}\left(Y, \mathcal{O}_{Y}\left(n A_{n}\right)\right)=\frac{1}{2}\left(\left(n A_{n}\right)^{2}\right)=n^{2}
$$

by the Riemann-Roch theorem. On the other hand,

$$
h^{1}\left(X, \mathcal{O}_{X}\left(C_{n}\right)\right) \geqslant n^{3}-3 n^{2}+3 n-1>c \cdot n^{2}
$$

for large enough $n$.

The surface $X$ studied above is of general type. It still could be true that Conjecture 2.5 .3 holds when restricted to rational surfaces, in any characteristic. For some evidence in this direction we now prove a particularly strong form of the conjecture in the case of smooth projective rational surfaces with an effective anticanonical divisor, and hence in particular for smooth projective toric surfaces.

Proposition 3.1.3 Let $X$ be a smooth projective rational surface having an effective anticanonical divisor $D \in\left|-K_{X}\right|$. Then there exists a constant $c_{X}$ such that for every prime divisor $C \subset X$ we have $h^{1}(X, C) \leqslant c_{X}$. In fact, the maximum value of $h^{1}(X, C)$ is either 0 or 1 , or it occurs when $C$ is a component of $D$.

Proof. Let $C$ be a prime divisor on $X$. Clearly, we can assume that $C$ is not a component of $D$. Thus $-K_{X} \cdot C=D \cdot C \geqslant 0$, and if $-K_{X} \cdot C=0$, then $C$ is disjoint from $D$.

Suppose $-K_{X} \cdot C>0$. If $C^{2} \geqslant 0$, then $h^{1}(X, C)=0$ by [22, Theorem III.1(a, b)]. If $C^{2}<0$, then $0>C^{2}=2 p_{a}(C)-2-K_{X} \cdot C>2 p_{a}(C)-2$ by adjunction, hence $p_{a}(C)=0$ and $C$ is smooth and rational with $C^{2}=-1$. Consider

$$
0 \rightarrow \mathcal{O}_{X} \rightarrow \mathcal{O}_{X}(C) \rightarrow \mathcal{O}_{C}(C) \rightarrow 0 .
$$

Since $X$ is rational we have $h^{2}\left(X, \mathcal{O}_{X}\right)=h^{1}\left(X, \mathcal{O}_{X}\right)=0$. Since $C$ is smooth and rational with $C^{2}=-1$, we have $h^{1}\left(C, \mathcal{O}_{C}(C)\right)=0$. Thus $h^{1}\left(X, \mathcal{O}_{X}(C)\right)=0$.

We are left with the case that $D \cdot C=-K_{X} \cdot C=0$, hence $C$ is disjoint from $D$. Thus $\mathcal{O}_{C}(C)=K_{C}$ by adjunction, so $h^{1}\left(C, \mathcal{O}_{C}(C)\right)=1$, and therefore from $(\star)$ we have $h^{1}(X, C)=1$.

\section{$3.2 \quad$ Speciality on blow-ups of $\mathbb{P}^{2}$}

In this section we look at the speciality of nef linear systems on blow-ups of $\mathbb{P}^{2}$. We try to formulate a statement, which is valid without assuming that the points we blow up are in general position. The examples presented here suggest that one needs to reformulate the problem stated in Section 2.3.

The first example shows that multiples of a special effective nef linear system might no longer be special. 
Example 3.2.1 This example is based on the existence of very ample but special linear systems. Let $p_{1}, \ldots, p_{25}$ be transversal intersection points of two smooth curves of degree 5 . Let $f: X \rightarrow \mathbb{P}^{2}$ be the blow-up of the plane at these 25 points. By [19, p. 796], for $1 \leqslant r \leqslant 2$ and any $m>0$, the linear system

$$
L=(5 m+r) H-m\left(E_{1}+\cdots+E_{25}\right),
$$

where $H$ is the class of the line and $E_{i}$ are the exceptional divisors of $f$, is very ample and special, but Serre vanishing implies that some multiple of $L$ is no longer special. Let $X^{\prime} \rightarrow X$ be the blow-up of $X$ at an additional point, and let $L^{\prime}$ be the pullback to $X^{\prime}$ of $L$. Then we have an example of a linear system $L^{\prime}$ which is special and nef but not ample and for which $s L^{\prime}$ is non-special for $s \gg 0$.

The second example shows that speciality might persist.

Example 3.2.2 Let $C$ and $D$ be smooth plane curves of degree $d \geqslant 3$ intersecting transversally in $p_{1}, \ldots, p_{d^{2}}$. Let $f: X \rightarrow \mathbb{P}^{2}$ be the blow-up of the plane at these points. The linear system

$$
L=d H-\sum_{i=1}^{d^{2}} E_{i}
$$

is special (again because its virtual dimension is negative). The same remains true for all multiples of $L$. This follows from the restriction sequence

$$
\left.0 \rightarrow m L \rightarrow(m+1) L \rightarrow(m+1) L\right|_{C} \rightarrow 0 .
$$

Indeed, by Serre duality we have $h^{2}(m L)=0$ for all $m \geqslant 1$. Also, as $L$ is a pencil of disjoint curves, we have $\left.L\right|_{C}=\mathcal{O}_{C}$. Then taking the long cohomology sequence of the restriction sequence we have the mapping

$$
\cdots \rightarrow H^{1}(X,(m+1) L) \rightarrow H^{1}\left(C, \mathcal{O}_{C}\right) \rightarrow 0 .
$$

The assumption $d \geqslant 3$ guarantees that $C$ is non-rational, hence $h^{1}\left(C, \mathcal{O}_{C}\right)=g(C)>$ 0 .

The next example shows that speciality may increase linearly while the number of global sections remains fixed.

Example 3.2.3 Let $C \subset \mathbb{P}^{2}$ be a quartic curve with a simple node at $p_{0}$ and smooth otherwise. Such a curve exists by a simple dimension count. Then we can take 12 points $p_{1}, \ldots, p_{12}$ on $C$ in such a way that $C$ is the unique quartic passing through $p_{0}$ with multiplicity 2 and through $p_{1}, \ldots, p_{12}$ with multiplicities all equal to 1 . Let $f: X \rightarrow \mathbb{P}^{2}$ be the blow-up of the plane at the points $p_{0}, \ldots, p_{12}$. We consider the linear system $L=4 H-2 E_{0}-E_{1}-\cdots-E_{12}$ on $X$. By a slight abuse of notation we write $C$ also for the proper transform of $C$ on $X$. It is a smooth curve of genus 2 . If the points $p_{1}, \ldots, p_{12}$ are generic enough, then $\left.m L\right|_{C}$ has no global sections for all $m \geqslant 1$ and by Riemann-Roch on $C$ we have $h^{1}\left(C,\left.m L\right|_{C}\right)=1$. Using again the restriction sequence we get

$$
h^{0}(X, m L)=1 \text { and } h^{1}(X, m L)=m \text { for all } m \geqslant 1 .
$$

These examples suggest the following problem. 
Problem 3.2.4 Let $X$ be the blow-up of $\mathbb{P}^{2}$ at $r$ distinct points ( $r$ is arbitrary and also the position of the points is arbitrary but we require the points to be distinct) and let $L$ be an effective nef divisor on $X$. Then is it true that either

a) there exists $m \geqslant 1$ such that $h^{1}(m L)=0$ (and this $m$ should be 1 if the points are in general position); or

b) there is a (not necessarily irreducible) curve $C$ on $X$ such that $|L-C| \neq \varnothing$, $p_{a}(C)>0$ and $L \cdot C=0$ ?

\subsection{Bounded negativity}

In the course of the discussions, we investigated the following problem, see [21, Conjecture 1.2.1].

Conjecture 3.3.1 (Bounded Negativity) Let $X$ be a smooth projective surface in characteristic zero. There exists a positive constant $b(X)$ bounding the selfintersection of reduced, irreducible curves on $X$, i.e.,

$$
C^{2} \geqslant-b(X)
$$

for every reduced, irreducible curve $C \subset X$.

The restriction to characteristic zero is in general essential (see Example 3.3.3) and of course so is the hypothesis that the curves be reduced, but it is not necessarily essential that they be irreducible. See Section 3.8 for further discussion.

One situation where Bounded Negativity holds is when the anti-canonical divisor is $\mathbb{Q}$-effective (see [21, I.2.3]):

Proposition 3.3.2 Let $X$ be a smooth projective surface such that for some integer $m>0$ the pluri-anti-canonical divisor $-m K_{X}$ is effective. Then there exists a positive constant $b(X)$ such that $C^{2} \geqslant-b(X)$ for every irreducible curve $C$ on $X$.

Proof. As $-m K_{X}$ is effective, there exist only finitely many irreducible curves $C$ such that $-K_{X} \cdot C<0$. Hence apart from these finitely many prime divisors, we have $-K_{X} \cdot C \geqslant 0$, in which case by the adjunction formula

$$
C^{2}=2 p_{a}-2-K_{X} \cdot C \geqslant 2 p_{a}-2 \geqslant-2 .
$$

Note that the hypothesis of $\mathbb{Q}$-effectivity holds for instance on toric surfaces. Another case where the conjecture is clearly true is when $-K_{X}$ is nef (with the same argument as that in the proof of Proposition 3.3.2).

The following example (see also [21, Remark 1.2.2]) shows that the restriction on the characteristic in Conjecture 3.3.1 cannot be avoided in general (but perhaps it can be avoided by restricting $X$ to be, for example, rational).

Example 3.3.3 (Exercise V.1.10, [25]) Let $C$ be a smooth curve of genus $g \geqslant 2$ defined over a field of characteristic $p>0$ and let $X$ be the product surface $X=$ $C \times C$. The graph $\Gamma_{q}$ of the Frobenius morphism defined by taking $q=p^{r}$-th powers is a smooth curve of genus $g$ and self-intersection $\Gamma_{q}^{2}=q(2-2 g)$. With $r$ going to infinity, we obtain a sequence of smooth curves of fixed genus with self-intersection going to minus infinity. 
In view of this example it is interesting to ask if at least one of the following is true.

Conjecture 3.3.4 (Weak Bounded Negativity) Let $X$ be a smooth projective surface in characteristic zero and let $g \geqslant 0$ be an integer. There exists a positive constant $b(X, g)$ bounding the self-intersection of curves of geometric genus $g$ on $X$, i.e.,

$$
C^{2} \geqslant-b(X, g)
$$

for every reduced, irreducible curve $C \subset X$ of geometric genus $g$ (i.e., the genus of the normalization of $C$ ).

Conjecture 3.3.5 (Very Weak Bounded Negativity) Let $X$ be a smooth projective surface in characteristic zero and let $g \geqslant 0$ be an integer. There exists a positive constant $b_{s}(X, g)$ bounding the self-intersection of smooth curves of geometric genus $g$ on $X$, i.e.,

$$
C^{2} \geqslant-b_{s}(X, g)
$$

for every irreducible smooth curve $C \subset X$ of geometric genus $g$.

It would be interesting to know if a bound of this type extends to a family of surfaces, i.e., if the following question has an affirmative answer.

Problem 3.3.6 Let $f: Y \rightarrow B$ be a morphism from a smooth projective threefold $Y$ to a smooth curve $B$ such that the general fibre is a smooth surface. Is there a constant $b(Y, g)$ such that

$$
C^{2} \geqslant-b(Y, g)
$$

for all vertical curves $C \subset Y$ (i.e., $f(C)=a$ point) of geometric genus $g$ ? (Here the self-intersection is computed in the fibre of $f$ containing $C$.)

We show that to a large extent both Conjectures 3.3 .4 and 3.3 .5 are true. The key ingredient of the proofs is the following vanishing result, [14, Corollary 6.9]. We recall the basic properties of log differential forms in Appendix A.

Theorem 3.3.7 (Bogomolov-Sommese Vanishing) Let $X$ be a smooth projective variety defined over an algebraically closed field of characteristic zero, $L$ a line bundle, $C$ a normal crossing divisor on $X$. Then

$$
H^{0}\left(X, \Omega_{X}^{a}(\log C) \otimes L^{-1}\right)=0
$$

for all $a<\kappa(X, L)$.

Corollary 3.3.8 Let $X$ be a smooth projective surface defined over an algebraically closed field of characteristic zero, $C$ a normal crossing divisor on $X$. Then $\Omega_{X}^{1}(\log C)$ contains no big line bundles.

Proof. Note that $L$ being big means $\kappa(X, L)=2$. An inclusion $L \hookrightarrow \Omega_{X}^{1}(\log C)$ gives rise to a non-trivial section of $H^{0}\left(X, \Omega_{X}^{1}(\log C) \otimes L^{-1}\right)$, which vanishes according to Theorem 3.3.7.

Remark 3.3.9 This kind of result was first observed by Bogomolov for the cotangent bundle of a surface itself:

$$
\text { If } L \subset \Omega_{X}^{1} \text { is a line bundle, then } L \text { is not big. }
$$

We refer to [54, Proposition 2.2] for a nice and detailed proof.

Remark 3.3.10 Theorem 3.3.7 and statement (2) are known to be false in positive characteristic, see [31, Remark 7.1]. 


\subsection{Partial proof of Conjecture 3.3.5; Very Weak Bounded Negativity}

Here we prove Conjecture 3.3 .5 on surfaces with Kodaira dimension $\kappa(X) \geqslant 0$.

Let $X$ be a surface as in Theorem 3.3.7 and let $\mathcal{F}$ be a coherent sheaf of rank $r$ on $X$. Recall that the discriminant $\Delta(\mathcal{F})$ is defined as

$$
\Delta(\mathcal{F}):=2 r c_{2}(\mathcal{F})-(r-1) c_{1}(\mathcal{F})^{2} .
$$

If $\mathcal{F}$ is a rank 2 vector bundle, then this reduces to

$$
\Delta(\mathcal{F})=4 c_{2}(\mathcal{F})-c_{1}(\mathcal{F})^{2}
$$

The interest in the discriminant of a vector bundle stems in part from the following useful numerical criterion for stability of vector bundles on surfaces. Recall first

Definition 3.4.1 Let $\mathcal{F}$ be a rank 2 vector bundle on a smooth projective surface $X$. We call $\mathcal{F}$ unstable if there exist line bundles $A$ and $B$ on $X$ and a finite subscheme $Z \subset X$ (possibly empty) such that the sequence

$$
0 \rightarrow A \rightarrow \mathcal{F} \rightarrow B \otimes \mathcal{I}_{Z} \rightarrow 0
$$

is exact and the $\mathbb{Q}$-divisor

$$
P:=A-\frac{1}{2} c_{1}(\mathcal{F})
$$

satisfies the conditions

$$
P^{2}>0 \text { and } P \cdot H>0
$$

for all ample divisors $H$ on $X$.

Remark 3.4.2 The conditions in the definition imply that $P$ is a big divisor.

The fundamental result of Bogomolov [5] is the following numerical criterion

Theorem 3.4.3 (Bogomolov) Let $\mathcal{F}$ be a rank 2 vector bundle on a smooth projective surface $X$. If $\Delta(\mathcal{F})<0$, then $\mathcal{F}$ is unstable.

As a corollary we prove Very Weak Bounded Negativity for smooth curves on surfaces with $\kappa(X) \geqslant 0$.

Proposition 3.4.4 Let $X$ be a smooth projective surface with $\kappa(X) \geqslant 0$ and let $C \subset X$ be a smooth curve of genus $g(C)$. Then

$$
C^{2} \geqslant c_{1}^{2}(X)-4 c_{2}(X)-4 g(C)+4 .
$$

Proof. We consider the following exact sequence which arises via an elementary transformation of $\Omega_{X}^{1}$ along $\Omega_{C}^{1}$ (see [29, Example 5.2.3])

$$
0 \rightarrow W:=\Omega_{X}^{1}(\log C) \otimes \mathcal{I}_{C} \rightarrow \Omega_{X}^{1} \rightarrow \Omega_{C}^{1} \rightarrow 0 .
$$

By [29, Proposition 5.2.2] we have

$$
c_{1}(W)=\left(K_{X}+C\right)-2 C=K_{X}-C \text { and } c_{2}(W)=c_{2}\left(\Omega_{X}^{1}\right)+\operatorname{deg}\left(\Omega_{C}^{1}\right)-K_{X} \cdot C .
$$

Hence

$$
\Delta(W)=4 c_{2}(X)-c_{1}^{2}(X)+4 g-4+C^{2} .
$$


If we assume that $C^{2}<c_{1}^{2}(X)-4 c_{2}(X)-4 g(C)+4$, then $\Delta(W)<0$ and $W$ is unstable. According to Definition 3.4.1 there exists then a line bundle $A \subset W$ such that

$$
A-\frac{1}{2} c_{1}(W)=A+\frac{1}{2}\left(C-K_{X}\right) \text { is big. }
$$

It follows that

$$
A+C \subset \Omega_{X}^{1}(\log C)
$$

and since $\kappa(X) \geqslant 0$ and $C$ is effective

$$
A+C=\left(A+\frac{1}{2} C-\frac{1}{2} K_{X}\right)+\frac{1}{2} K_{X}+\frac{1}{2} C
$$

is big as well. However, this contradicts the Bogomolov-Sommese Vanishing, Theorem 3.3 .7

Remark 3.4.5 Note that a statement as in the above Proposition cannot hold on ruled surfaces, i.e., there is no lower bound on $C^{2}$ with $C$ smooth depending only on the genus of $C$ and Chern numbers of $X$. Indeed, all Hirzebruch surfaces $F_{n}=\mathbb{P}\left(\mathcal{O}_{\mathbb{P}^{1}} \oplus \mathcal{O}_{\mathbb{P}^{1}}(-n)\right)$ have the same invariants $c_{1}^{2}\left(F_{n}\right)=8, c_{2}\left(F_{n}\right)=4$ and each $F_{n}$ contains a smooth rational curve of self-intersection $-n$. This observation is of course not a counterexample either to Conjecture 3.3.1 or to Conjecture 3.3.5. It merely means that the bound for $C^{2}$ on ruled surfaces must depend on something else. A reasonable possibility, in the case where $X$ is obtained by blowing up $r$ points of a surface $Y$ when $b(Y)$ exists, is to try to show that $b(X)$ can be defined in terms of $b(Y)$ and $r$.

\subsection{Partial proof of Conjecture 3.3.4: Weak Bounded Negativity}

The main auxiliary ingredient in this part is the logarithmic version of the MiyaokaYau inequality.

Theorem 3.5.1 (Logarithmic Miyaoka-Yau inequality) Let $X$ be a smooth projective surface and $C$ a smooth curve on $X$ such that the adjoint line bundle $K_{X}+C$ is $\mathbb{Q}$-effective, i.e., there is an integer $m>0$ such that $h^{0}\left(m\left(K_{X}+C\right)\right)>0$. Then

$$
c_{1}^{2}\left(\Omega_{X}^{1}(\log C)\right) \leqslant 3 c_{2}\left(\Omega_{X}^{1}(\log C)\right),
$$

equivalently $\left(K_{X}+C\right)^{2} \leqslant 3\left(c_{2}(X)-2+2 g(C)\right)$.

We refer to Appendix $\mathrm{A}$ for the proof. Note that our statement of this inequality is not the most general, but it suffices for our needs. We also need the following elementary lemma.

Lemma 3.5.2 Let $X$ be a smooth projective surface, $C \subset X$ a reduced, irreducible curve of geometric genus $g(C), P \in C$ a point with mult $_{P} C \geqslant 2$. Let $\sigma: \widetilde{X} \rightarrow X$ be the blow-up of $X$ at $P$ with the exceptional divisor $E$. Let $\widetilde{C}=\sigma^{*}(C)-m E$ be the proper transform of $C$. Then the inequality

$$
\widetilde{C}^{2} \geqslant c_{1}^{2}(\widetilde{X})-3 c_{2}(\widetilde{X})+2-2 g(\widetilde{C})
$$

implies

$$
C^{2} \geqslant c_{1}^{2}(X)-3 c_{2}(X)+2-2 g(C)
$$


Proof. We have

$$
C^{2}=\widetilde{C}^{2}+m^{2}, \quad c_{1}^{2}(X)=c_{1}^{2}(\widetilde{X})+1, \quad c_{2}(X)=c_{2}(\widetilde{X})-1 \text { and } g(C)=g(\widetilde{C}) .
$$

Hence

$$
\begin{aligned}
C^{2} & =m^{2}+\widetilde{C}^{2} \\
& \geqslant m^{2}+c_{1}^{2}(\widetilde{X})-3 c_{2}(\widetilde{X})+2-2 g(\widetilde{C}) \\
& =m^{2}+c_{1}^{2}(X)-1-3 c_{2}(X)-3+2-2 g(C) \\
& \geqslant c_{1}^{2}(X)-3 c_{2}(X)+2-2 g(C) .
\end{aligned}
$$

Proposition 3.5.3 Let $X$ be a smooth projective surface with $\kappa(X) \geqslant 0$. Then for every reduced, irreducible curve $C \subset X$ of geometric genus $g(C)$ we have

$$
C^{2} \geqslant c_{1}^{2}(X)-3 c_{2}(X)+2-2 g(C) .
$$

Proof. The idea is to reduce the statement to a smooth curve and use Theorem 3.5.1,

We blow up $f: \widetilde{X}=X_{N} \rightarrow X_{N-1} \rightarrow \ldots \rightarrow X_{0}=X$ resolving step-by-step the singularities of $C$. The proper transform of $C$ in $\widetilde{X}$ is then a smooth irreducible curve $\widetilde{C}$. Applying Lemma 3.5 .2 recursively to every step in the resolution $f$ we see that it is enough to prove inequality (3) for $C$ smooth.

This follows easily from the Logarithmic Miyaoka-Yau inequality 3.5.1. Note that our assumption $\kappa(X) \geqslant 0$ implies that $K_{\widetilde{X}}+\widetilde{C}$ is $\mathbb{Q}$-effective. Hence

$c_{1}^{2}(X)+2 C \cdot\left(K_{X}+C\right)-C^{2}=c_{1}^{2}\left(\Omega_{X}^{1}(\log C)\right) \leqslant 3 c_{2}\left(\Omega_{X}^{1}(\log C)\right)=3 c_{2}(X)-6+6 g(C)$.

By adjunction $C \cdot\left(K_{X}+C\right)=2 g(C)-2$ and rearranging terms we arrive at (3) .

Remark 3.5.4 Note that Proposition 3.5.3 applies in particular to smooth curves and provides in general a better bound than that in Proposition 3.4.4. We do not pursue the optimality problem here, and we found it instructive to provide two possible proofs for the Very Weak Bounded Negativity Conjecture.

\subsection{Bounded negativity conjecture and Seshadri constants}

We next point out an interesting connection between bounded negativity and a question on Seshadri constants posed by Demailly in [10, Question 6.9]:

Problem 3.6.1 Is the global Seshadri constant

$$
\varepsilon(X):=\inf \{\varepsilon(L) \mid L \in \operatorname{Pic}(X) \text { ample }\}
$$

positive for every smooth projective surface $X$ ?

At present, this is unknown. In fact, it is unknown whether for every fixed $x \in X$ the quantity

$$
\varepsilon(X, x)=\inf \{\varepsilon(L, x) \mid L \in \operatorname{Pic}(X) \text { ample }\}
$$

is always positive. The latter, however, would be a consequence of the Bounded Negativity Conjecture: 
Proposition 3.6.2 If the Bounded Negativity Conjecture is true, then

$$
\varepsilon(X, x)>0
$$

for every smooth projective surface $X$ in characteristic zero, and every $x \in X$.

The proof below actually gives an effective lower bound on $\varepsilon(X, x)$ : If $Y=$ $\mathrm{Bl}_{x}(X)$ is the blow-up of $X$ at $x$, then

$$
\varepsilon(X, x) \geqslant \frac{1}{\sqrt{b(Y)+1}}
$$

So if we knew that the constant $b(Y)$ is the same for every one-point blow-up of $X$ (or at least bounded from below by a constant that is independent of $x$ ), then we would get a lower bound on $\varepsilon(X)$.

Proof of the proposition. Let $C \subset X$ be an irreducible curve of multiplicity $m$ at $x$, and let $\widetilde{C} \subset Y$ be its proper transform on the blow-up $Y$ of $X$ in $x$. Then

$$
C^{2}-m^{2}=\left(f^{*} C-m E\right)^{2}=\widetilde{C}^{2} \geqslant-b(Y) .
$$

Consider first the case where $m \leqslant \sqrt{b(Y)}$. Then

$$
\frac{L \cdot C}{m} \geqslant \frac{L \cdot C}{\sqrt{b(Y)}} \geqslant \frac{1}{\sqrt{b(Y)}}
$$

In the alternative case, where $m>\sqrt{b(Y)}$, we have

$$
C^{2} \geqslant m^{2}-b(Y)>0
$$

and hence, using the Index Theorem, we get

$$
\frac{L \cdot C}{m} \geqslant \frac{\sqrt{L^{2}} \sqrt{C^{2}}}{m} \geqslant \sqrt{1-\frac{b(Y)}{m^{2}}} \geqslant \sqrt{1-\frac{b(Y)}{b(Y)+1}}=\frac{1}{\sqrt{b(Y)+1}} .
$$

An alternative argument for the proof of the proposition goes as follows. Suppose that $\varepsilon(X, x)=0$. Then there is a sequence of ample line bundles $L_{n}$ and curves $C_{n}$ such that

$$
\frac{L_{n} \cdot C_{n}}{m_{n}} \rightarrow 0
$$

where $m_{n}$ denotes the multiplicity of $C_{n}$ at $x$. We use now that the blow-up $Y=$ $\mathrm{Bl}_{x}(X)$ has bounded negativity, so that for the proper transform $\widetilde{C}_{n}$ of $C_{n}$ we have

$$
\left(\widetilde{C}_{n}\right)^{2} \geqslant-b(Y)^{2} .
$$

But $\left(\widetilde{C}_{n}\right)^{2}=\left(f^{*} C_{n}-m_{n} E\right)^{2}=C_{n}^{2}-m_{n}^{2}$, which, upon using the Index Theorem, tells us that

$$
m_{n}^{2}-b(Y) \leqslant C_{n}^{2} \leqslant \frac{\left(L_{n} \cdot C_{n}\right)^{2}}{L_{n}^{2}} .
$$

So we see that

$$
1-\frac{b(Y)}{m_{n}^{2}} \leqslant \frac{C_{n}^{2}}{m_{n}^{2}} \leqslant \frac{\left(L_{n} \cdot C_{n}\right)^{2}}{m_{n}^{2}} \cdot \frac{1}{L_{n}^{2}} \leqslant\left(\frac{L_{n} \cdot C_{n}}{m_{n}}\right)^{2} .
$$

Now, the left hand side of this chain of inequalities tends to one, whereas the right hand side goes to zero, a contradiction. 


\subsection{The Weighted Bounded Negativity Conjecture}

The following conjecture is yet another variant of the Bounded Negativity Conjecture 3.3.1.

Conjecture 3.7.1 (Weighted Bounded Negativity) Let $X$ be a smooth projective surface in characteristic zero. There exists a positive constant $b_{w}(X)$ such that

$$
C^{2} \geqslant-b_{w}(X)(H \cdot C)^{2}
$$

for every irreducible curve $C \subset X$ and every big and nef line bundle $H$ satisfying $H \cdot C>0$.

The interest in this conjecture is due to the fact that this conjecture is sufficient to imply the conclusion of the previous proposition, by the following result.

Proposition 3.7.2 If the Weighted Bounded Negativity Conjecture is true, then

$$
\varepsilon(X, x)>0
$$

for every smooth projective surface $X$ in characteristic zero, and every $x \in X$.

Proof. As above, there are two proofs, and one of these yields the effective lower bound

$$
\varepsilon(X, x) \geqslant \frac{1}{\sqrt{b_{w}(Y)+1}}
$$

where $Y=\mathrm{Bl}_{x}(X)$ is the blow-up of $X$ at $x$. We first look at this proof.

Let $C \subset X$ be an irreducible curve of multiplicity $m$ at $x$ and $L$ an ample line bundle on $X$. Let $\widetilde{C} \subset Y$ be the proper transform of $C$ on $Y$. Since $f^{*} L$ is big and nef and $f^{*} L \cdot \widetilde{C}=L \cdot C>0$, the Weighted Bounded Negativity Conjecture on $Y$ yields the existence of a constant $b_{w}(Y)$ such that

$$
C^{2}-m^{2}=\left(f^{*} C-m E\right)^{2}=(\widetilde{C})^{2} \geqslant-b_{w}(Y)\left(f^{*} L \cdot \widetilde{C}\right)^{2}=b_{w}(Y)(L \cdot C)^{2} .
$$

Then, using the Index Theorem, we obtain

$$
\frac{(L \cdot C)^{2}}{m^{2}} \geqslant \frac{L^{2} C^{2}}{m^{2}} \geqslant L^{2}\left(1-b_{w}(Y) \frac{(L \cdot C)^{2}}{m^{2}}\right),
$$

that is,

$$
\left(\frac{L \cdot C}{m}\right)^{2}\left(1+b_{w}(Y) L^{2}\right) \geqslant L^{2}
$$

This yields

$$
\frac{L \cdot C}{m} \geqslant \sqrt{\frac{L^{2}}{1+b_{w}(Y) L^{2}}}=\sqrt{\frac{1}{\frac{1}{L^{2}}+b_{w}(Y)}} \geqslant \sqrt{\frac{1}{1+b_{w}(Y)}}=\frac{1}{\sqrt{1+b_{w}(Y)}},
$$

as asserted.

The second version of the proof goes as follows: Suppose that $\varepsilon(X, x)=0$. Then there is a sequence of ample line bundles $L_{n}$ and curves $C_{n}$ such that

$$
\frac{L_{n} \cdot C_{n}}{m_{n}} \rightarrow 0
$$


where $m_{n}$ denotes the multiplicity of $C_{n}$ at $x$. Let $f: Y=\mathrm{Bl}_{x}(X) \rightarrow X$ be the blow-up of $X$ at $x$ and denote by $\widetilde{C}_{n}$ the proper transform of $C_{n}$. Since $f^{*} L_{n}$ is big and nef and $f^{*} L_{n} \cdot \widetilde{C}_{n}=L_{n} \cdot C_{n}>0$, the Weighted Bounded Negativity Conjecture on $Y$ yields the existence of a constant $b_{w}(Y)$ such that

$$
\left(\widetilde{C}_{n}\right)^{2} \geqslant-b_{w}(Y)\left(f^{*} L_{n} \cdot \widetilde{C}_{n}\right)^{2}=-b_{w}(Y)\left(L_{n} \cdot C_{n}\right)^{2} .
$$

But $\left(\widetilde{C}_{n}\right)^{2}=\left(f^{*} C_{n}-m_{n} E\right)^{2}=C_{n}^{2}-m_{n}^{2}$, which yields

$$
\frac{C_{n}^{2}}{m_{n}^{2}}-1 \geqslant-b_{w}(Y) \frac{\left(L_{n} \cdot C_{n}\right)^{2}}{m_{n}^{2}} .
$$

Combining with the Index Theorem, we obtain

$$
1-b_{w}(Y)\left(\frac{L_{n} \cdot C_{n}}{m_{n}}\right)^{2} \leqslant \frac{C_{n}^{2}}{m_{n}^{2}} \leqslant\left(\frac{L_{n} \cdot C_{n}}{m_{n}}\right)^{2} \cdot \frac{1}{L_{n}^{2}} .
$$

But the left hand side of this chain of inequalities tends to one, whereas the right hand side tends to zero, a contradiction.

Note that the second proof does not give an effective lower bound for $\varepsilon(X, x)$.

\subsection{Bounded negativity for reducible curves}

We now consider a reducible version of Conjecture 3.3.1;

Conjecture 3.8.1 (Reducible Bounded Negativity) Let $X$ be a smooth projective surface in characteristic zero. Then there exists a positive constant $b^{\prime}(X)$ bounding the self-intersection of reduced curves on $X$, i.e.,

$$
C^{2} \geqslant-b^{\prime}(X)
$$

for every reduced (but not necessarily irreducible) curve $C \subset X$.

Conjecture 3.3.1 implies Conjecture 3.8.1 in a very explicit way.

Proposition 3.8.2 Let $X$ be a smooth projective surface (in any characteristic) for which there is a constant $b(X)$ such that $C^{2} \geqslant-b(X)$ for every reduced, irreducible curve $C \subset X$. Then $C^{2} \geqslant-\rho(X) b(X)\lceil b(X) / 2\rceil$ for every reduced curve $C \subset X$, where $\rho(X)$ is the Picard number of $X$ (i.e., the rank of the Néron-Severi group $\mathrm{NS}(X)$ of $X)$.

Proof. Let $C=C_{1}+\cdots+C_{r}$ be a sum of distinct curves $C_{i}$ on $X$. By reindexing we may write this sum as $C=\sum_{i j} C_{i j}$, where for each $i$, the sets $\left\{C_{i j}\right\}_{j}$ are linearly independent in the Néron-Severi group, and for $i<i^{\prime}$, the span of $\left\{C_{i j}\right\}_{j}$ contains the span of $\left\{C_{i^{\prime} j}\right\}_{j}$.

For each $i$, let $B_{i}=\sum_{j} C_{i j}$ and let $\beta$ be the number of elements $B_{i}$. Then, $B_{i}^{2} \geqslant \sum_{j} C_{i j}^{2} \geqslant-\rho(X) b(X)$, where the first inequality is because all of the cross terms are non-negative, and the second because $C_{i j}^{2} \geqslant-b(X)$ but there can be at most $\rho(X)$ linearly independent elements in the Néron-Severi group.

Also note for any distinct prime divisors $A_{1}, \ldots, A_{n}, A$ such that the $A_{i}$ are linearly independent in $\mathrm{NS}(X)$ and $A^{2}<0$ with $A$ in the span (in $\mathrm{NS}(X)$ ) of the $A_{i}$, we have $A \cdot A_{i}>0$ for some $i$, and hence $\left(A_{1}+\cdots+A_{n}\right) \cdot A>0$. This is because we 
can write $A+\sum_{s} a_{s} A_{s}=\sum_{t} a_{t} A_{t}$ for non-negative rational coefficients $a_{s}$ and $a_{t}$, with the sums over $s$ and $t$ running over disjoint subsets of $\{1, \ldots, n\}$. Since $A \neq A_{i}$ for all $i$, we have $A \cdot A_{i} \geqslant 0$ for all $i$, but if $A \cdot A_{i}=0$ for all $i$, then we would have $0>A^{2}=A \cdot\left(A+\sum_{s} a_{s} A_{s}\right)=A \cdot\left(\sum_{t} a_{t} A_{t}\right)=0$. In particular this shows for $i<i^{\prime}$ that $B_{i} \cdot C_{i^{\prime} j}>0$.

Let $u=\min (\beta,\lceil b(X) / 2\rceil)$. We will now show that $C^{2} \geqslant\left(B_{1}+\cdots+B_{u}\right)^{2}$. The result follows from this, since $\left(B_{1}+\cdots+B_{u}\right)^{2} \geqslant B_{1}^{2}+\cdots+B_{u}^{2} \geqslant-u \rho(X) b(X) \geqslant$ $-\rho(X) b(X)\lceil b(X) / 2\rceil$. If $u=\beta$, then $C=B_{1}+\cdots+B_{u}$, and clearly $C^{2} \geqslant\left(B_{1}+\right.$ $\left.\cdots+B_{u}\right)^{2}$. Otherwise, $C=B_{1}+\cdots+B_{u}+D_{1}+\cdots+D_{t}$, where $D_{1}, \ldots, D_{t}$ are the terms of the sum $C=C_{1}+\cdots+C_{r}$ not already subsumed by $B_{1}+\cdots+B_{u}$. But $C^{2} \geqslant\left(B_{1}+\cdots+B_{u}\right)^{2}+\sum_{i} 2\left(B_{1}+\cdots+B_{u}\right) \cdot D_{i}+\sum_{i} D_{i}^{2}$, and $B_{i} \cdot D_{j}>0$ for each $i$ and $j$ (by our observation above regarding $B_{i} \cdot C_{i^{\prime} j}>0$ ), so $\sum_{i} 2\left(B_{1}+\cdots+B_{u}\right.$ ). $D_{i}+\sum_{i} D_{i}^{2} \geqslant \sum_{i}\left(2 u+D_{i}^{2}\right) \geqslant \sum_{i}(2 u-b(X)) \geqslant 0$. Thus $C^{2} \geqslant\left(B_{1}+\cdots+B_{u}\right)^{2}$, as claimed.

It seems unlikely that the bound given in Proposition 3.8.2 is ever sharp. For a blow-up $X$ of $\mathbb{P}^{2}$ at $n$ generic points, we expect by the SHGH Conjecture that $C^{2} \geqslant$ -1 for any reduced, irreducible curve $C \subset X$. The bound given in Proposition 3.8.2 would then be $C^{2} \geqslant-(n+1)$ for reduced but possibly reducible curves $C$. It is easy to produce reduced examples with $C^{2}=-n$; for example, take $C=E_{1}+\cdots+E_{n}$. On the other hand, one cannot reach $C^{2}=-(n+1)$. Indeed, the only possibility would be to have $n+1$ disjoint $(-1)$ curves. As the Picard number of the blow-up is $(n+1)$, this would contradict the Index Theorem.

Thus it is of interest to give specific examples of reduced curves with $C^{2}$ as negative as possible. Clearly the negativity of $C^{2}$ can grow with $\rho(X)$, so it makes sense to normalize $C^{2}$. We will consider several examples involving blow-ups of $\mathbb{P}^{2}$ at $n$ points, with the goal of finding values of $C^{2} / n$ that are as negative as possible.

Example 3.8.3 Let $X$ be the blow-up of $\mathbb{P}^{2}$ at $n$ collinear points, and let $C$ be the proper transform of the line containing the points. Then $C^{2} / n$ is approximately -1 . Here $C$ is a prime divisor.

Example 3.8.4 Consider a general map $f: \mathbb{P}^{1} \rightarrow \mathbb{P}^{2}$ of degree $d$. Let $C \subset X$ be the proper transform of the image of $\mathbb{P}^{1}$ (which has degree $d$ ) where $X$ is obtained by blowing up the singular points of $f\left(\mathbb{P}^{1}\right)$ (there are $n=\left(\begin{array}{c}d-1 \\ 2\end{array}\right)$ nodes). Thus $C^{2} / n$ is approximately -2 . Here $C$ is again a prime divisor.

Example 3.8.5 Let $X$ be the blow-up of the points of intersection of $s>2$ general lines in $\mathbb{P}^{2}$; thus $n=\left(\begin{array}{l}s \\ 2\end{array}\right)$. Let $C$ be the proper transform of the union of the lines. Then $C^{2}=s(s-2)$, so $C^{2} / n$ is approximately -2 .

Example 3.8.6 Assume the ground field $k$ is algebraically closed of characteristic $p$. Let $q$ be some power of $p>0$. Blow up the points of $\mathbb{P}^{2}$ with coordinates in the finite field $\mathbb{F}_{q}$. Let $C$ be the proper transform of the union of all lines through pairs of the points blown up. There are $n=q^{2}+q+1$ points, and also $q^{2}+q+1$ lines, and $q+1$ lines pass through each of the points. Thus $C^{2}=\left(q^{2}+q+1\right)^{2}-(q+1)^{2}\left(q^{2}+q+1\right)=$ $-q\left(q^{2}+q+1\right)$, so $C^{2} / n=-q$, which is approximately $-\sqrt{n}$.

Remark 3.8.7 This last example raises the question of how negative $C^{2}$ can be if $C$ is the proper transform of a union of lines. This makes contact with interesting problems studied by combinatorists. For example, let $S \subset \mathbb{P}^{2}$ be a set of $n$ distinct 
points, and let $\Lambda=\left\{L_{1}, \ldots, L_{l}\right\}$ be a set of $l$ distinct lines. Let $M(S, \Lambda)$ be the incidence matrix; its rows correspond to the points, its columns correspond to the lines, and an entry is either 1 or 0 according to whether the corresponding point lies or does not lie on the corresponding line. Let $|M|$ be the sum of the entries of $M$.

If $X$ is obtained by blowing up the points of $S$, and $C=L_{1}^{\prime}+\cdots+L_{l}^{\prime}$, where $L_{i}^{\prime}$ is the proper transform of $L_{i}$, then $C^{2} \geqslant \sum_{i}\left(L_{i}^{\prime}\right)^{2}=l-|M|$, with equality if $S$ contains all of the points where any two of the lines meet.

It's easy to get a coarse bound on $C^{2} / n$. Clearly, $l \leqslant\left(\begin{array}{l}n \\ 2\end{array}\right)$, and $|M| \leqslant l n$, so $C^{2} / n \geqslant\left(\begin{array}{l}n \\ 2\end{array}\right)(1-n) / n \geqslant-\left(\begin{array}{l}n \\ 2\end{array}\right)$. For points with real coefficients and lines defined over the reals, the Szemerédi-Trotter Theorem [51] gives an order of magnitude estimate $|M| \leqslant O\left((l n)^{2 / 3}+l+n\right)$. There also are Szemerédi-Trotter type results over finite fields; for example, see Bourgain-Katz-Tao [6] and Vinh [55].

\subsection{Imposing higher vanishing order at one point}

We next study the polynomial interpolation problem on the projective plane. We denote by $\mathcal{L}\left(d ; m_{1}, m_{2}, \ldots, m_{n}\right)$ the linear system of homogeneous polynomials of degree $d$ passing through $n$ generic points with multiplicities at least $m_{1}, \ldots, m_{n}$. We want to study $\mathcal{L}\left(d ; m_{1}+1, m_{2}, \ldots, m_{n}\right)$, provided $\mathcal{L}\left(d ; m_{1}, m_{2}, \ldots, m_{n}\right) \neq \varnothing$.

Let $\pi: X \rightarrow \mathbb{P}^{2}$ the blow-up of $n$ generic points; let $E_{i}=\pi^{-1}\left(p_{i}\right)$ be the exceptional divisors and $H=\pi^{*} L$. Consider the divisor $D=d H-\sum m_{i} E_{i}$. We can identify

$$
\mathcal{L}\left(d ; m_{1}, m_{2}, \ldots, m_{n}\right)=H^{0}\left(\mathcal{O}_{X}(D)\right)
$$

and

$$
\mathcal{L}\left(d ; m_{1}+1, m_{2}, \ldots, m_{n}\right)=H^{0}\left(\mathcal{O}_{X}\left(D-E_{1}\right)\right) .
$$

Thus, the starting point for our problem is to analyze the cohomology of the exact sequence

$$
0 \rightarrow \mathcal{O}_{X}\left(D-E_{1}\right) \rightarrow \mathcal{O}_{X}(D) \rightarrow \mathcal{O}_{D}\left(D_{\mid E_{1}}\right) \rightarrow 0 .
$$

As a matter of fact, passing to the long exact cohomology sequence we have

$$
\begin{aligned}
0 & \rightarrow H^{0}\left(\mathcal{O}\left(D-E_{1}\right)\right) \rightarrow H^{0}(\mathcal{O}(D)) \stackrel{\rho}{\rightarrow} H^{0}\left(\mathcal{O}\left(D_{\mid E_{1}}\right)\right) \\
\rightarrow H^{1}\left(\mathcal{O}\left(D-E_{1}\right)\right) & \rightarrow H^{1}(\mathcal{O}(D)) \rightarrow 0
\end{aligned}
$$

The image of $\rho$ is a linear system on $E_{1}$ of degree $m_{1}$.

Example 3.9.1 Consider $D=4 H-E_{1}-2 E_{2}-\cdots-2 E_{5}$, then

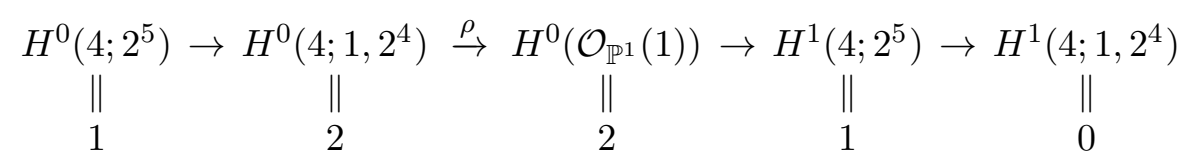

It is known that $\mathcal{L}\left(4 ; 2^{5}\right)$ is a special system, since its expected dimension is -1 but there is an element in it: the quartic $2 C$, where $C$ is the conic passing through the five points. Thus $H^{1}\left(4 ; 2^{5}\right)=1$, and in this case $\rho$ does not have maximal rank.

Example 3.9.2 As a slight modification of the above example we next consider $D=6 H-2 E_{1}-3 E_{2}-\cdots-3 E_{5}$. Then 


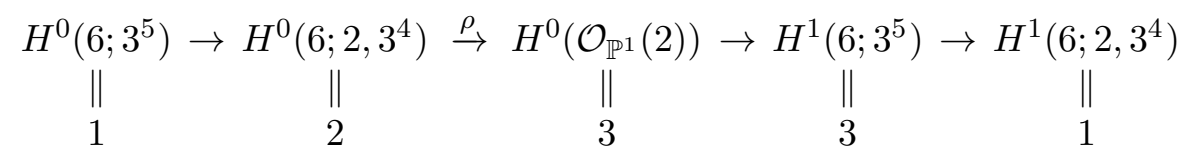

This shows that also in this case the rank of $\rho$ is not maximal.

The above examples give hints about the behavior of $\mathcal{L}\left(d ; m_{1}+1, m_{2}, \ldots, m_{n}\right)$, given $\mathcal{L}\left(d ; m_{1}, m_{2}, \ldots, m_{n}\right) \neq \varnothing$. We formulate the following:

Conjecture 3.9.3 Let $X$ be the blow-up of $\mathbb{P}^{2}$ in $r$ general points, $D$ an effective divisor on $X$ and $E$ a (-1)-curve. For the restriction map $|D| \stackrel{\rho}{\rightarrow}\left|D_{\mid E}\right|$ one has:

1. All base points in the image of $\rho$ come from a base curve in $|D|$.

2. If $|D|$ has no fixed component, then $\rho$ has maximal rank.

If points in special position are allowed, then it is not hard to find counterexamples to this statement. However, we would like to consider the analogous question when $E$ is a $(-n)$-curve, with $n>1$. We denote by $\mathcal{L}\left(d ; \overline{m_{1}, \ldots, m_{r}}, m_{r+1}, \ldots, m_{n}\right)$ the linear system of curves of degree $d$ with points of multiplicities $m_{1}, \ldots, m_{n}$, the first $r$ of which are collinear, but which are otherwise general. The discussions led to the following problem:

Problem 3.9.4 Is it true that, if $d \geqslant m_{1}+\cdots+m_{r}$, then $\mathcal{L}\left(d ; m_{1}, \ldots, m_{n}\right) \neq \varnothing$ if and only if $\mathcal{L}\left(d ; \overline{m_{1}, \ldots, m_{r}}, m_{r+1}, \ldots, m_{n}\right) \neq \varnothing$ ?

This statement seems quite strong. If true, the induction arguments common in approaches to the SHGH conjecture as [26], [7, [15] would be significantly simplified, which might even lead to a proof. However we want to remark that some genericity condition is necessary here as well; in particular it is not possible to allow the existence of many negative curves even if they meet the system non-negatively. For example, if we pick four general lines, and four general points on each of them, there is a quartic, if not a pencil, through the 16 points, which is not the case for general points. It is easy to see that we can generalize this counterexample just considering $d>3$ lines and $d$ points on each line. We don't have, at the moment, any counterexample to the statement with only two or three lines.

\subsection{Geometrization of Dumnicki's method [12]}

Let $D \subset \mathbb{N}^{2}$ be a finite set, such that $D=P \cap \mathbb{N}^{2}$, where $P$ is a convex polygon with integer vertices. We denote by $\mathcal{L}(D)$ the linear series on the affine plane $\mathbb{A}^{2}$ spanned by monomials in $D$ (we identify a point $(k, l) \in \mathbb{N}^{2}$ with the monomial $\left.x^{k} y^{l}\right)$. It can also be viewed as the complete linear series associated to the polarized toric variety $\left(X_{D}, L_{D}\right)$ defined by the polygon $D$, i.e., $\mathcal{L}(D)=\mathbb{P}\left(\left|L_{D}\right|\right)$. We also write $\mathcal{L}\left(D, m_{1}, \ldots, m_{r}\right)_{p_{1}, \ldots, p_{r}}$ for the subsystem of $\mathcal{L}(D)$ consisting of polynomials vanishing at the given $r$ smooth points with multiplicities at least $m_{1}, \ldots, m_{r}$. One then defines:

$$
\mathcal{L}\left(D, m_{1}, \ldots, m_{r}\right)=\min _{p_{1}, \ldots, p_{r} \in X_{D}}\left\{\mathcal{L}\left(D, m_{1}, \ldots, m_{r}\right)_{p_{1}, \ldots, p_{r}}\right\}
$$


Consider a partition $D=D_{1} \cup D_{2} \cup \cdots \cup D_{r}$ determined by lines (not through the vertices). Then the following holds:

Fact 1: If $\mathcal{L}\left(D_{i}, m_{i}\right)=\varnothing$, for all $i=1, \ldots, r$, then $\mathcal{L}\left(D, m_{1}, \ldots, m_{r}\right)=\varnothing$.

Fact 2: Given $D \subset \mathbb{N}^{2}$ such that $|D|=\left(\begin{array}{c}m+1 \\ 2\end{array}\right)$, then $\mathcal{L}(D, m) \neq \varnothing$ if and only if there exists a nonzero polynomial $F \in \mathbb{Q}[x, y]$ with $\operatorname{deg}(F)=m-1$, such that $F(a, b)=0$ for all $(a, b) \in D$.

From Fact 2 (and Bézout's Theorem), we immediately obtain the following

Fact 3: If, for $D \subset \mathbb{N}^{2}$, there exist $m$ horizontal (resp. vertical) lines $\ell_{1}, \ldots, \ell_{m}$ such that

$$
D \subset \bigcup_{k=1}^{m} \ell_{k}, \quad \#\left(D \cap \ell_{k}\right) \leqslant k \text { for } k=1, \ldots, m
$$

then $\mathcal{L}(D ; m)=\varnothing$.

As an example, we prove that the divisor $13 H-5 E_{1}-4 E_{2}-\cdots-4 E_{10}$ is not semi-effective (see Definition 2.2.1 and Problem 2.2.2). For a fixed $n$, we consider the set $D=\left\{(x, y) \in \mathbb{N}^{2}: x+y \leqslant 13 n\right\} \subset \mathbb{N}^{2}$. We will cut $D$ with nine lines into ten subsets $D_{1}, \ldots, D_{10}$. The equations of lines are given by the following functions (for small $\varepsilon>0$ ):

$$
\begin{array}{ll}
f_{1}: & -x-y+5 n-1+\varepsilon \\
f_{2}: & x-9 n-1+\varepsilon \\
f_{3}: & y-9 n-1+\varepsilon \\
f_{4}: & x-y-5 n-1+\varepsilon \\
f_{5}: & -x+y-5 n-1+\varepsilon \\
f_{6}: & 3 x-y-15 n-1+\varepsilon \\
f_{7}: & -3 x+y+3 n-1+\varepsilon \\
f_{8}: & (2 n+1) x-2 n y-2 n^{2}-5 n-1+\varepsilon \\
f_{9}: & x+3 y-21 n+\varepsilon .
\end{array}
$$

The sets $D_{1}, \ldots, D_{10}$ are defined inductively by

$$
D_{j}=\left(D \backslash\left(D_{1} \cup \cdots \cup D_{j-1}\right)\right) \cap\left\{(x, y): f_{j}(x, y)>0\right\}, \quad \text { for } j=1, \ldots, 9,
$$

and $D_{10}=D \backslash\left(D_{1} \cup \cdots \cup D_{9}\right)$. Due to Fact 1 , it is enough to show that $\mathcal{L}\left(D_{1} ; 5 n\right)=\varnothing$ and $\mathcal{L}\left(D_{k} ; 4 n\right)=\varnothing$ for $k=2, \ldots, 10$. For each $D_{k}$ we will proceed using Fact 3 , and this is more or less a straightforward computation. We present it for $D_{1}$ (for $D_{2}, \ldots, D_{5}$ it is very similar) and $D_{6}$ (for $D_{7}, \ldots, D_{10}$ it is also very similar).

The set $D_{1}$ is given by equations $-x-y+5 n-1+\varepsilon>0, x \geqslant 0, y \geqslant 0$ and it is in fact a simplex (triangle) with $5 n$ lattice points along the bottom border line, so the assumptions of Fact 3 are satisfied.

The set $D_{6}$ is given by $3 x-y-15 n-1+\varepsilon>0, x+y-13 n \leqslant 0, x-y-5 n-1+\varepsilon<0$. Let $(x, y) \in D_{6}$. Observe that for $x>9 n$ we would have $y<4 n$, so $-y>-4 n$ and $x-y-5 n-1>9 n-4 n-5 n-1>-1$, hence $x-y-5 n-1 \geqslant 0$, a contradiction. For $x \leqslant 5 n$ we would have $y \leqslant 3 x-15 n-1 \leqslant-1$, a contradiction. Hence $x \in[5 n+1,9 n]$, so $D_{6}$ lies on at most $4 n$ vertical lines. From the the first and the last defining inequality we easily obtain that for $(x, y) \in D_{6}, x \in[5 n+1,7 n]$ we must have $y \in[x-5 n, 3 x-15 n-1]$. But for $x=5 n+1$ we have exactly two lattice points in the interval $[x-5 n, 3 x-15 n-1]=[1,2]$. If $x$ increases by one then $\#[x-5 n, 3 x-15 n-1]$ inceases by two, so we have at most $2,4, \ldots, 4 n-2,4 n$ points on $2 n$ vertical lines $\ell_{2}=\{x=5 n+1\}, \ell_{4}=\{x=5 n+2\}, \ldots, \ell_{4 n}=\{x=7 n\}$. Similarly 


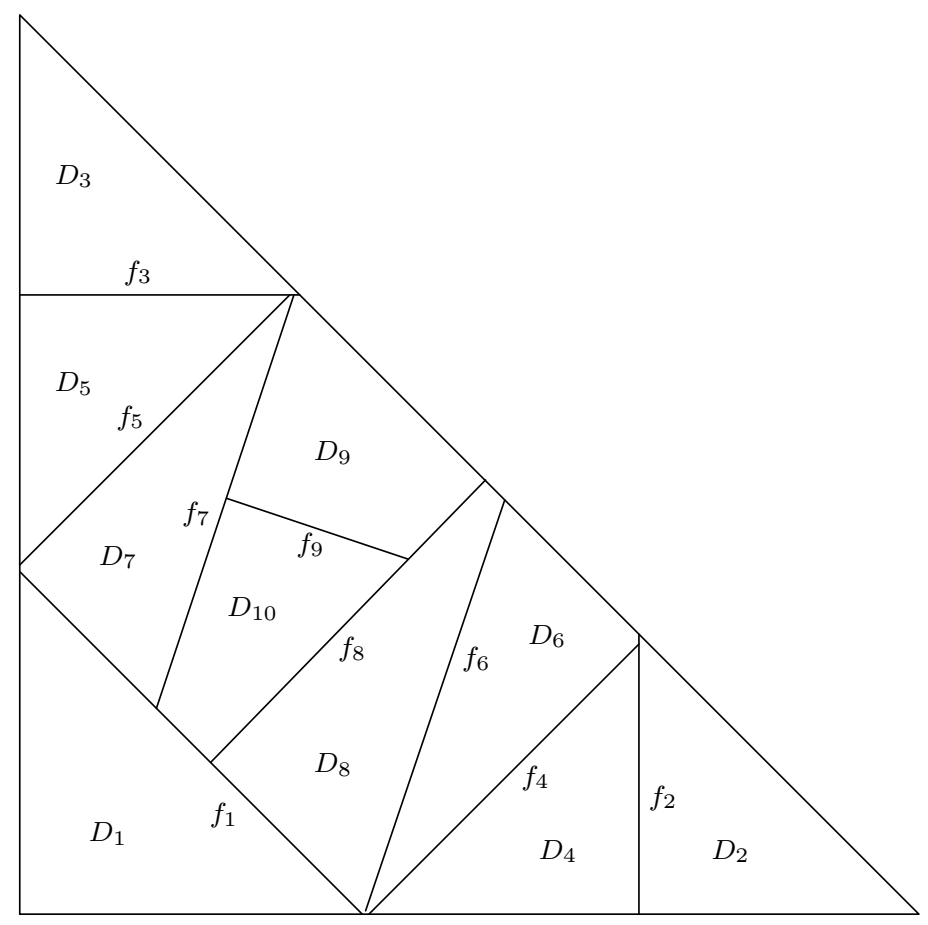

Figure 1: Subdivision of $D$

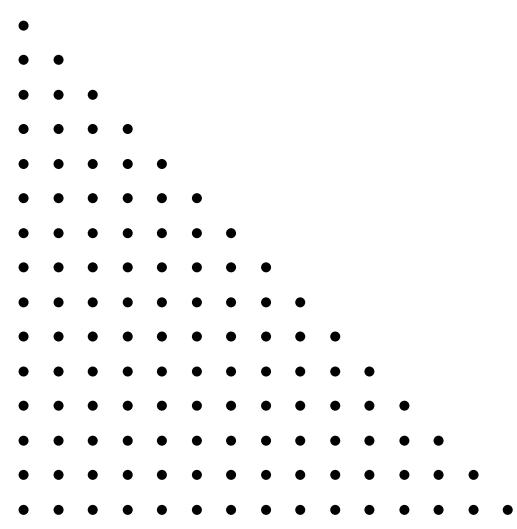

Figure 2: The set $D_{1}$ for $n=3$

we show that on lines $\ell_{4 n-1}=\{x=7 n+1\}, \ell_{4 n-3}=\{x=7 n+2\}, \ldots, \ell_{1}=\{x=9 n\}$ we have at most $4 n-1,4 n-3, \ldots, 1$ points.

Facts 1 and 2 can be understood from the point of view of toric degenerations, and as a result we can get a more geometric proof of non-semi-effectivity of $13 L-$ $5 E_{1}-4 E_{2}-\cdots-4 E_{10}$. The translation of Fact 1 into a toric statement is based on the construction of a projective toric variety from any polytope $D$ (defined as an intersection of half-spaces) in a space $M$ as follows.

Let $Q$ be a face of $D$. Define a cone $C_{Q}$ in the dual space $N$ by

$$
C_{Q}=\{v \in N \mid\langle v, p-q\rangle \geqslant 0 \text { for all } p \in D \text { and } q \in Q\}
$$

As $Q$ varies over all of the faces of $D$, one gets a fan of cones $F_{D}$, which defines 


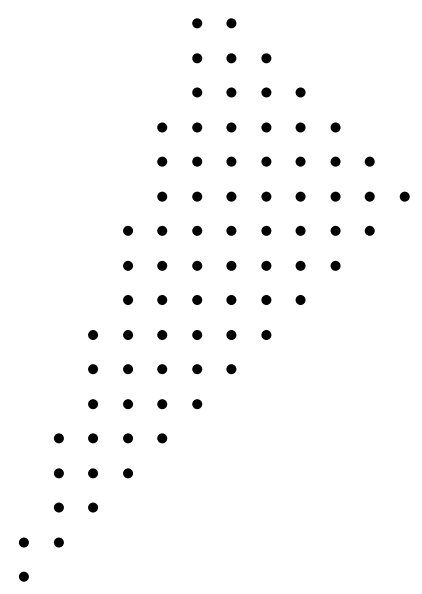

Figure 3: The set $D_{6}$ for $n=3$

a toric variety $X_{D}$ (see [18, Section 1.5). Furthermore, this toric variety comes equipped with an ample line bundle $L_{D}$ (see [18], Section 3.4, page 72).

For example, if the polytope is the interval $[0, d]$ in one-dimensional space, then the variety is the projective line, and the line bundle has degree $d$. Similarly, if the polytope is the usual triangle with vertices $(0,0),(0, d),(d, 0)$ in the plane, then the variety is the projective plane and the line bundle has degree $d$.

The construction works up to a point with unbounded polytopes, too. The one that is useful for us is to take a polytope $D$ and cross it with the positive reals. This gives a new polytope, $D^{\prime}$, and the associated toric variety is $X_{D} \times \mathbb{A}^{1}$, the product of the original toric variety for $D$ with the affine line. The line bundle is just the pullback of the line bundle on $X_{D}$.

A more interesting example of an unbounded polytope can be used to see a degeneration. Take the set of three line segments: $(0,0)$ to $(1,-1) ;(1,-1)$ to $(2,-1)$; and $(2,-1)$ to $(3,0)$. Take as the (unbounded) polytope all points $(x, y)$ which lie on or above these line segments:

$$
D=\left\{\begin{array}{lll} 
& \text { if } 0 \leqslant x \leqslant 1 & \text { then } y \geqslant-x ; \\
(x, y) \mid 0 \leqslant x \leqslant 3 ; & \text { if } 1 \leqslant x \leqslant 2 & \text { then } y \geqslant-1 ; \\
& \text { if } 2 \leqslant x \leqslant 3 & \text { then } y \geqslant x-3
\end{array}\right\}
$$

Each of the four vertices of $D$ gives four maximal cones of the fan in the dual space, and these are bounded by the rays $(-1,0)$ and $(-1,1) ;(-1,1)$ and $(0,1) ;(0,1)$ and $(1,1) ;(1,1)$ and $(1,0)$. The associated toric variety is the blow-up of $\mathbb{P}^{1} \times \mathbb{A}^{1}$ at two points of the central fiber, giving a degeneration of $\mathbb{P}^{1}$ to a chain of three $\mathbb{P}^{1}$ ss. As for the line bundle, it restricts to degree 3 on the general fiber, and degree one on each component of the special fiber. This then realizes the degeneration of the twisted cubic curve to a chain of three lines.

What we see in this example is the 3 -Veronese of $\mathbb{P}^{1}$, defined by the polytope which is just the interval $[0,3]$, and a 'subdivision' of the interval suitably used to create the degeneration. In order to properly construct the degeneration, we had to create the 'lifting' of the subdivision to one dimension higher.

In general, one may in fact use a similar construction. One needs a similar lifting of the subdivision of the 'bottom' faces of an unbounded polytope. The key ingredient is to have that the faces of the unbounded polytope lie exactly above the 
sub-polytopes. This is the idea of a regular subdivision.

Suppose $D$ is a polytope, and $S=\bigcup D_{i}$ is a subdivision of $D$. Suppose that we have a real-valued continuous function $F$ on $D$, which is linear on each $D_{i}$. One says that the function $F$ is strictly $S$-convex if it is a convex function, with the additional property that for any subpolytope $D_{i}$ of $S$, if $L_{i}$ is the linear function for the subpolytope, extended to the entire space, then $F(p)>L_{i}(p)$ for all $p$ in $D \backslash D_{i}$.

A subdivision $S$ of $D$ is called regular if $D$ has a strictly $S$-convex function. One may consult [57], Chapter 5, for additional detail on these ideas.

Now suppose we have a polytope $D$ in $\mathbb{R}^{n}$, defining a toric variety $X_{D}$, as above. Suppose we have a subdivision $S$ of $D$, which is regular; let $F$ be a strictly $S$-convex function on $D$. Define the unbounded polytope $P(F)$ in $\mathbb{R}^{n+1}$ by:

$$
P(F)=\left\{(x, y) \in P \times \mathbb{R}^{1} \mid y \geqslant F(x)\right\}
$$

This polytope $P(F)$ defines a toric variety $Y$, which is a suitable blow-up of $X_{D} \times \mathbb{A}^{1}$ in the central fiber, and exhibits a degeneration of $X_{D}$ to a union of toric varieties defined by the subpolytopes $D_{i}$.

There is a more elementary way of seeing the degeneration, embedded in projective space. Suppose that $D$ is a polygon (with lattice points for vertices of course) whose set of lattice points is $\left\{m_{i}\right\}$. These lattice points correspond to monomials in the variables in the usual way: the lattice coordinates are the exponents of the monomials. If there are $k+1$ monomials, this defines a mapping $g: \mathbb{P}^{2} \rightarrow \mathbb{P}^{k}$ by sending a point $[x]$ to the point $\left[x^{m_{i}}\right]$, and $X_{D}$ is the image of this mapping.

Now suppose we have this subdivision $S$, with a strictly $S$-convex function $F$ as above. If $y$ is a coordinate on $\mathbb{A}^{1}$, this data defines a new mapping

$$
G: \mathbb{P}^{2} \times\left(\mathbb{A}^{1}-0\right) \rightarrow \mathbb{P}^{k}
$$

by sending a pair $([x], y)$ to the point $\left[y^{F\left(m_{i}\right)} x^{m_{i}}\right]$.

For a fixed $y$ not equal to zero, this is simply a scaling of the original map $g$, and so the $\mathbb{P}^{2}$ fibers are all mapped to $X_{D}$. We want to know what happens in the limit as $y$ approaches zero. Of course, the values of $F$ may well be either negative, or all positive, and so we cannot take the limit with the above formula.

To see what happens, consider one of the subpolytopes $D_{i}$, and the corresponding linear function $L=L_{i}$. Vary the above formula by multiplying through by $y^{-L}$. This will have the effect that as $y$ approaches zero, a limiting value will be available in the projective space.

The simplest example of this construction is to take a polytope $D$ and divide it into two pieces, by a hyperplane. Suppose that this hyperplane does not pass through any of the lattice points. Then one can start with a convex function $H$ that is linear on the two sides of the hyperplane. Form the construction above, and then take the convex hull of the graph of $H$, using the lattice points. This will produce two 'primary' subpolytopes as faces of this convex hull, containing the two subsets of the lattice points, on the two sides of the hyperplane. It will also contain a set of 'secondary' subpolytopes that cross the boundary of the hyperplane.

The construction then produces a toric degeneration, into two 'primary' toric varieties, and a set of 'secondary' ones. If we want to use this construction to understand an interpolation problem à la Dumnicki, we put the limits of the fat points on the two 'primary' varieties, and none on the secondary ones. If the interpolation problems on the two 'primary' varieties give empty linear systems, then the linear system will be empty in the limit, and therefore empty on the general fiber (by semicontinuity). 
In Dumnicki's example, with the triangle subdivided into ten subpolytopes, one easily sees that this subdivision can be achieved iteratively, by making a singlehyperplane subdivision, nine times. This should give the result, and provide a 'toric' interpretation for the example.

The proof of Fact 2 mainly involves an interpolation matrix $M(D)$. Let $\left(X_{D}, L_{D}\right)$ be the polarized toric surface defined by the polygon $D$. Recall that $X_{D}=\cup_{v \in \operatorname{Vert}(D)} U_{v}$ where $U_{v}$ denotes an affine neighborhood around the fixed point on $X_{D}$ corresponding to $v$. By placing one vertex of the polytope at 0 one chooses an affine patch around the corresponding fixed point so that:

$$
H^{0}\left(U_{v},\left.L_{D}\right|_{U_{v}}\right)=\bigoplus_{\alpha=(a, b) \in D} \mathbb{A}\left\langle s_{\alpha}\right\rangle, \text { where } s_{\alpha}=x^{a} y^{b}
$$

Let $J_{m}(D)=J_{m}\left(L_{D}\right)$ be the $m^{\text {th }}$ jet sheaf, i.e., the coherent sheaf defined locally at smooth points $p$, as $J_{m}\left(L_{D}\right)_{p}=H^{0}\left(L_{D} \otimes \mathcal{O}_{X_{D}} / \mathfrak{m}_{p}^{m+1}\right)$, where $\mathfrak{m}_{p}$ denotes the maximal ideal at $p$. The $m^{\text {th }}$ evaluation map at a smooth point $p$ is defined as the map:

$$
\nu_{k, p}: H^{0}\left(X_{D}, L_{D}\right) \rightarrow J_{m}\left(L_{D}\right)_{p}
$$

that assigns $\nu_{k, p}\left(s_{i}\right)=\left(s_{i}(p), \ldots, \frac{\partial^{i+j} s_{i}}{\partial x^{j} \partial x^{i}}(p), \ldots\right)_{1 \leqslant i+j \leqslant m}$ to a basic section $s_{i}$, for a choice of local coordinates $(x, y)$ in a neighborhood $U_{v}$ around $p$. The map is in fact independent of the choice of $U_{v}$ and $\operatorname{Im}\left(\nu_{k, p}\right)=\mathbb{O}_{p}^{m}$ is referred to as the $m^{\text {th }}$ osculating space at $p$.

Recall that the dimension of $\mathbb{O}_{p}^{m}$ at a generic smooth point, e.g., at the generic point in the torus $T \cong\left(\mathbb{C}^{*}\right)^{2} \subset X_{D}$, is constant and it is called the generic osculation dimension.

For a smooth point $p$ the dimension is:

$$
\operatorname{dim}\left(\mathcal{L}(D, m)_{p}\right)=\operatorname{dim}\left(X_{D}, L_{D}\right)-\operatorname{dim}\left(\mathbb{O}_{p}^{m-1}\right) .
$$

We denote by $1=(1,1)$ the generic point of the torus. Because $\operatorname{dim}\left(\mathbb{O}_{1}^{m}\right) \geqslant \operatorname{dim}\left(\mathbb{O}_{p}^{m}\right)$ for all other smooth points $p$,

$$
\begin{gathered}
\operatorname{dim}(\mathcal{L}(D, m))=\operatorname{dim}\left(\mathcal{L}(D, m)_{1}\right) \text { and } \\
\mathcal{L}(D, m)=\varnothing \text { if and only if } \operatorname{dim} \mathbb{O}_{1}^{m-1}=\operatorname{dim}\left(X_{D}, L_{D}\right) .
\end{gathered}
$$

Let $\operatorname{dim}\left(H^{0}\left(X_{D}, L_{D}\right)\right)=|D|=N$. The map $\nu_{m-1,1}: H^{0}\left(X_{D}, L_{D}\right) \rightarrow J_{m-1}\left(L_{D}\right)_{1}$ is represented by the $N \times\left(\begin{array}{c}m+1 \\ 2\end{array}\right)$ matrix $M(D)$, whose rows are indexed by the derivatives, until order $m-1$, with respect to $x$ and $y$, of the $s_{i}$ evaluated at 1 while the columns are indexed by monomials in $D$. Thus, for example, the $(i, j)$ entry in $M(D)$ is the derivative, in row $i$, of the monomial in column $j$.

$$
M(D)=\left(\begin{array}{cccc}
1 & 1 & \ldots & 1 \\
a_{1} & a_{2} & \ldots & a_{N} \\
b_{1} & b_{2} & \ldots & b_{N} \\
a_{1} b_{1} & a_{2} b_{2} & \ldots & a_{N} b_{N} \\
\ldots & \ldots & \ldots & \ldots
\end{array}\right)
$$

where $D=\left\{\left(a_{1}, b_{1}\right), \ldots,\left(a_{N}, b_{N}\right)\right\}$.

In the case when $|D|=\left(\begin{array}{c}m+1 \\ 2\end{array}\right)$ the matrix is a square matrix and thus one has $\mathcal{L}(D, m)=\varnothing$ if and only if $\operatorname{det}(M(D)) \neq 0$, from which the existence of a polynomial $F \in \mathbb{Q}[x, y]$ with $\operatorname{deg}(F)=m-1$, such that $F(a, b)=0$ for all $(a, b) \in D$ is derived. 
It is important to remark here that the proof gives an interpretation of the lattice points given by $D$ as points in $\mathbb{A}^{2}$.

We observed that the set of derivatives used can be substituted by any set $E=$ $\left\{\partial_{x}^{\alpha_{1}} \partial_{y}^{\beta_{1}}, \ldots, \partial_{x}^{\alpha_{N}} \partial_{y}^{\beta_{N}}\right\}$ which (interpreting $\left(\alpha_{i}, \beta_{i}\right)$ as points in $\left.\mathbb{N}^{2}\right)$ is closed under downward and leftward moves; in the literature such sets $E$ are known as staircases (see [15]) and they are used to define monomial ideals $I(E)=\left(x^{\alpha} y^{\beta}\right)_{(\alpha, \beta) \notin E}$. In the method above one then has to use a modified jet sheaf where $\mathfrak{m}_{p}^{m+1}$ is replaced by the translation $I(E)_{p}$ of $I(E)$ to $p$ by the action of the torus. Denoting by $\mathcal{L}(D, E)$ the subsystem of $\mathcal{L}(D)$ consisting of sections which belong to the monomial ideal $\mathcal{I}(E)_{1}$ supported at the generic point, we obtain a

Generalized Fact 2: Given $D \subset \mathbb{N}^{2}$ such that $|D|=|E|$, then $\mathcal{L}(D, E) \neq \varnothing$ if and only if there exists a nonzero polynomial $F \in \mathbb{Q}[x, y]$ containing only polynomials of $E$, such that $F(a, b)=0$ for all $(a, b) \in D$.

Such a generalization allows to deal with the Hermite interpolation schemes of tree type considered by Lorentz, (see [37, Section 3), which is actually a specialized case of the general monomial interpolation problem suggested as Problem 2.4.2. Note that in the original problem the coordinates in which the scheme is monomial can be "deformed" by the immersion of the scheme in the surface, whereas here they are torically fixed. Thus when $\mathcal{L}(D, E)=\varnothing$ it follows that $\mathcal{L}\left(D, \mathcal{I}_{Y}\right)=\varnothing$ for general schemes $Y$ isomorphic to $\operatorname{Spec} k[x, y] / I_{E}$, but not conversely.

\subsection{Linear systems connected to hyperplane arrangements}

We keep the notation introduced in Section 2.7. Results of Falk-Yuzvinsky [16] show that certain combinatorial objects known as weak $(k, m)$-multinets give rise to components of $R^{1}(\mathcal{A})$, and [45] shows that a weak $(k, m)$-multinet corresponds to a divisor $A$ on $X$ with $h^{0}(A)=2$. If the weak multinet is actually a net, then $D_{\mathcal{A}}=A+B$, with $h^{0}(B) \geqslant k m-\left(\begin{array}{c}m+1 \\ 2\end{array}\right)$. This decomposition then gives rise to determinantal equations (and syzygies) in the Orlik-Terao ideal. In particular, since $h^{0}(A)=2$, if $h^{0}(B)=m$, then $I$ contains the two by two minors of a matrix of linear forms, which has an Eagon-Northcott resolution, which has a very explicit description. The next example shows that not all linear first syzygies arise from components of $R^{1}(\mathcal{A})$.

Example 3.11.1 The arrangement below is obtained by deleting a line from the Maclane arrangement (Example 10.7 of [49]).

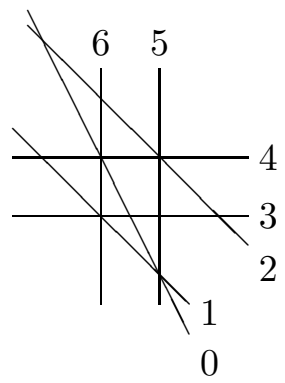

Figure 4: The $M_{8}^{-}$arrangement 
The graded betti numbers for the Orlik-Terao algebra are

\begin{tabular}{r|llllll} 
total & 1 & 8 & 36 & 56 & 35 & 8 \\
\hline 0 & 1 & - & - & - & - & \\
1 & - & 7 & 1 & - & - & - \\
2 & - & 1 & 35 & 56 & 35 & 8
\end{tabular}

The entry in position $(i, j)$ is $\operatorname{dim}_{\mathbb{C}} T o r_{i}^{R}(C(\mathcal{A}), \mathbb{C})_{i+j}$, so there are seven quadratic generators for $I$, and a single linear first syzygy. On the other hand, for this arrangement, $R^{1}(\mathcal{A})$ consists only of local components.

An analysis shows that we may choose a basis for the quadratic component $I_{2}$ so that the linear first syzygy involves only five of the seven minimal quadratic generators. Letting $J$ denote the ideal generated by these five elements, we compute that $J$ has betti diagram

\begin{tabular}{r|cllll} 
total & 1 & 5 & 12 & 10 & 2 \\
\hline 0 & 1 & - & - & - & - \\
1 & - & 5 & 1 & - & - \\
2 & - & - & 11 & 10 & 1 \\
3 & - & - & - & - & 1
\end{tabular}

The corresponding variety is a Cohen-Macaulay surface; intersecting with a generic $\mathbb{P}^{5}$ yields a smooth, projectively normal curve of genus seven and degree eleven. This curve appears in [48] as a counterexample to several conjectures in algebraic geometry. A sole linear first syzygy cannot arise from a decomposition of $D_{\mathcal{A}}$ : such a decomposition would yield at least one additional linear first syzygy.

\subsection{Limitations of multiplier ideal approach to bounds for symbolic powers}

It is natural to ask whether the strategy of [13], using asymptotic multiplier ideals, can be used to prove Conjecture 2.8.1 or any of the improved versions in Problem 2.8.2, or whether another strategy is needed. A weaker version of 2.8.1 was shown by Takagi-Yoshida using tight closure methods [52], but (as observed in [53]) the same result follows from the asymptotic multiplier ideal approach of [13]. On the other hand it seems doubtful that the same approach can prove Conjecture 2.8.1 in full strength. So, can the asymptotic multiplier ideals approach prove any of the statements in Problem 2.8.2, or at least weaker versions? We will see an example suggesting that the answer to this question is negative.

The strategy of $[13]$ is as follows. Let $I$ be an ideal with $\operatorname{bight}(I)=e$. Consider the graded system of ideals $I^{(\bullet)}=\left\{I^{(p)}\right\}_{p \in \mathbb{N}}$, the sequence of symbolic powers of $I$. For each positive real number $t$ there is an ideal associated to this graded system, called the $t^{\text {th }}$ asymptotic multiplier ideal of $I^{(\bullet)}$, and denoted $\mathcal{J}\left(t \cdot I^{(\bullet)}\right)$. These ideals enjoy a number of remarkable properties; see [13] (where they were introduced) or [35]. In particular it is shown in [13] that the following containments hold:

$$
I^{(r e)} \subseteq \mathcal{J}\left(r e \cdot I^{(\bullet)}\right) \subseteq \mathcal{J}\left(e \cdot I^{(\bullet)}\right)^{r} \subseteq I^{r} .
$$

Here the first and third inclusions follow more or less directly from the definition of multiplier ideals, while the second inclusion follows from the subadditivity theorem 
of Demailly-Ein-Lazarsfeld [11, 35]. The weak version of 2.8.1 follows from the fact that if $\mathcal{J}\left(\ell \cdot I^{(\bullet)}\right)=(1)$, which holds for sufficiently small $\ell$, then $I^{(r e-\ell)} \subseteq$ $\mathcal{J}\left(r e \cdot I^{(\bullet)}\right)$. (Conjecture 2.8.1 would follow if $\ell=e-1$.) Note that the second and third containments remain the same; only the first is made tighter.

To give a positive answer to Problem 2.8.2 by this approach would require an improvement at the far right-hand side of the above containments. An easy example suggests this may not be possible. Let $R=\mathbb{C}[x, y, z]$ and let $I=(x y, x z, y z)$, the ideal defining the union of the three coordinate axes (in particular $e=\operatorname{bight}(I)=2$ ). In general multiplier ideals are difficult to compute, and few examples are known; the situation is worse for asymptotic multiplier ideals, where even fewer examples have been computed. However, because this ideal $I$ is a monomial ideal, it is possible to compute the asymptotic multiplier ideals appearing in the above containments. This is carried out in [53] (following [39]) with the result that, for each $r$,

$$
\mathcal{J}\left(2 \cdot I^{(\bullet)}\right)^{r}=I^{r} .
$$

That is, it is not possible to improve the third containment while leaving the first and second the same.

It is still possible that an improvement might be attainable for some ideals, just not this particular one. However at this point the asymptotic multiplier ideals approach does not seem promising.

\section{A Logarithmic differentials and the Miyaoka-Yau inequality}

\section{A.1 Basics}

Here we provide a very quick overview of logarithmic differentials, in particular, no proofs are given. For more complete discussions the reader should consult [14] or [30], for example.

Let $X$ be a smooth projective variety of dimension $n$ over the complex numbers. A reduced divisor $D=D_{1}+\cdots+D_{r}$ is called a simple normal crossing divisor if all of its irreducible components are smooth, and every point of the support of $D$ has an open neighborhood $U$ sucht that $D$ restricted to $U$ looks like an intersection of coordinate hyperplanes. In particular, if $D$ is prime, then it is forced to be smooth.

With $(X, D)$ as above, let $x \in D$, and $D_{1}, \ldots, D_{s}$ be the irreducible components of $D$ containing the point $x$. By definition there exists an open neighborhood $x \in$ $U \subseteq X$, and a local coordinate system $x_{1}, \ldots, x_{n}$ at $x$ on $U$ such that

$$
D_{i} \cap U=V\left(x_{i}\right)
$$

for all $1 \leqslant i \leqslant s$. We call such a coordinate system a logarithmic coordinate system at $x$ along $D$.

In what follows, $D$ denotes a simple normal crossing divisor. At first we look at the following heuristic picture. We will look at various interesting subbundles inside the tangent bundle $T_{X}$ of $X$. In order to give some geometric intuition, we will often switch to the language of differential geometry.

With notation as above,

$$
T_{X} \otimes \mathcal{I}_{D / X} \subseteq T_{X}
$$

is a sub-vector bundle, that can be identified with vector fields on $X$ fixing $D$ pointwise. On the other hand, it is natural to consider the subbundle corresponding to 
vector fields that stabilize the divisor $D$. This latter is denoted by $T_{X}(-\log D)$, and is again a sub-vector bundle of $T_{X}$. Summarizing, one has a sequence of inclusions of vector bundles

$$
T_{X} \otimes \mathcal{I}_{D / X} \subseteq T_{X}(-\log D) \subseteq T_{X} .
$$

With the help of a little homological algebra one obtains a dual sequence of inclusions

$$
\left(T_{X}\right)^{*} \subseteq\left(T_{X}(-\log D)\right)^{*} \subseteq\left(T_{X} \otimes \mathcal{I}_{D / X}\right)^{*}
$$

Observe that

$$
\left(T_{X}\right)^{*} \simeq \Omega_{X}^{1},\left(T_{X} \otimes \mathcal{I}_{D / X}\right)^{*} \simeq \Omega_{X}^{1} \otimes \mathcal{O}_{X}(D) .
$$

In fact, we can write

$$
\Omega_{X}^{1} \subseteq \Omega_{X}^{1}(\log D) \subseteq \Omega_{X}^{1} \otimes \mathcal{O}_{X}(D),
$$

where $\left(T_{X}(-\log D)\right)^{*} \simeq \Omega_{X}^{1}(\log D)$, and the latter is called the sheaf of logarithmic differentials with poles along $D$. The formal definition is as follows.

Definition A.1.1 With notation as above, the sheaf of logarithmic differentials with poles along $D, \Omega_{X}^{1}(\log D)$, is the $\mathcal{O}_{X}$-submodule of $\Omega_{X}^{1} \otimes_{\mathcal{O}_{X}} \mathcal{O}_{X}(D)$ determined uniquely by the following properties:

1. $\left.\Omega_{X}^{1}(\log D)\right|_{X-D} \simeq \Omega_{X-D}^{1}$,

2. Let $U \subseteq X$ be an open subset intersecting D. For an element $f \in\left(\Omega_{X}^{1} \otimes\right.$ $\left.\mathcal{O}_{X}(D)\right)(U)$,

$$
f \in \Omega_{X}^{1}(\log D)(U) \text { if and only if } f_{x}=\sum_{i=1}^{s} g_{i} \frac{d x_{i}}{x_{i}}+\sum_{i=s+1}^{n} h_{i} d x_{i}
$$

for every (closed) point $x \in D \cap U$, and every logarithmic coordinate system $x_{1}, \ldots, x_{n}$ at $x$ along $D$, where $g_{i}, h_{i} \in \mathcal{O}_{X, x}$.

Next, we set

$$
\Omega_{X}^{p}(\log D):=\wedge^{p} \Omega_{X}^{1}(\log D)
$$

Remark A.1.2 For a smooth projective variety $X$ of dimension $n$ and a simple normal crossing divisor $D$ on $X$ we have the duality

$$
\left(\Omega^{j}(\log D)\right)^{*}=\Omega^{n-j}(\log D) \otimes \mathcal{O}_{X}\left(-K_{X}-D\right) .
$$

In particular, for a surface $X$,

$$
\left(\Omega_{X}^{1}(\log C)\right)^{*} \simeq \Omega_{X}^{1}(\log C) \otimes \mathcal{O}_{X}\left(-K_{X}-C\right),
$$

hence also

$$
\left(\operatorname{Sym}^{m} \Omega_{X}^{1}(\log C)\right)^{*} \simeq \operatorname{Sym}^{m} \Omega_{X}^{1}(\log C) \otimes \mathcal{O}_{X}\left(-m\left(K_{X}+C\right)\right) .
$$

Here $C \subseteq X$ denotes a simple normal crossing curve on the surface $X$. 
One of the fundamental tools for computing with logarithmic differentials is the collection of short exact sequences

$$
0 \rightarrow \Omega_{X}^{p} \rightarrow \Omega_{X}^{p}(\log D) \rightarrow \Omega_{D}^{p-1} \rightarrow 0
$$

for all $p \geqslant 1$, in particular,

$$
0 \rightarrow \Omega_{X}^{1} \rightarrow \Omega_{X}^{1}(\log D) \rightarrow \mathcal{O}_{D} \rightarrow 0
$$

From now on we assume that $X$ is a smooth projective surface and $C \subseteq X$ is an irreducible curve. We will compute the Chern classes of the bundle $\mathcal{F}:=\Omega_{X}^{1}(\log C)$. Since $\operatorname{rank} \mathcal{F}=2$, we only care about $c_{1}$ and $c_{2}$. From the Whitney sum formula [17, Theorem $3.2(\mathrm{e})]$ and the exact sequence (4) we obtain

$$
c_{t}\left(\Omega_{X}^{1}(\log C)\right)=c_{t}\left(\Omega_{X}^{1}\right) \cdot c_{t}\left(\mathcal{O}_{C}\right)
$$

and from the short exact sequence

$$
0 \rightarrow \mathcal{O}_{X}(-C) \rightarrow \mathcal{O}_{X} \rightarrow \mathcal{O}_{C} \rightarrow 0
$$

it follows that

$$
c_{t}\left(\mathcal{O}_{C}\right)=\frac{1}{c_{t}\left(\mathcal{O}_{X}(-C)\right)}=c_{t}\left(\mathcal{O}_{X}(C)\right)
$$

Hence

$$
\begin{aligned}
& 1+c_{1}\left(\Omega_{X}^{1}(\log C)\right) t+c_{2}\left(\Omega_{X}^{1}(\log C)\right) t^{2} \\
& \quad=\left(1+c_{1}\left(\Omega_{X}^{1}\right) t+c_{2}\left(\Omega_{X}^{1}\right) t^{2}\right) \cdot\left(1+c_{1}\left(\mathcal{O}_{X}(C)\right) t\right)
\end{aligned}
$$

which gives

$$
\begin{aligned}
& c_{1}\left(\Omega_{X}^{1}(\log C)\right)=c_{1}\left(\mathcal{O}_{X}(C)\right)+c_{1}\left(\Omega_{X}^{1}\right)=K_{X}+C \\
& c_{2}\left(\Omega_{X}^{1}(\log C)\right)=c_{2}\left(\Omega_{X}^{1}\right)+c_{1}\left(\Omega_{X}^{1}\right) \cdot c_{1}\left(\mathcal{O}_{X}(C)\right)=c_{2}(X)+\left(K_{X} \cdot C\right) .
\end{aligned}
$$

When it comes to computing various expressions in terms of Chern classes, we can identify $c_{1}\left(\mathcal{O}_{X}(C)\right)$ with $C$ and $c_{1}\left(\Omega_{X}^{1}\right)$ with $K_{X}$.

\section{A.2 The Miyaoka-Yau inequality for $\Omega_{X}^{1}(\log C)$}

The purpose of this section is to establish the logarithmic version of the MiyaokaYau inequality originally proved by Sakai [44, Theorem 7.6]. Sakai's argument, as will our exposition, follows closely that of Miyaoka in [38. Since it is our goal to provide a clear picture, we will present a reasonably self-contained proof. The main result is presented in Theorem A.2.8.

In the course of this section $X$ denotes an arbitrary smooth complex projective surface unless otherwise stated. We always work over the complex numbers. Our starting point is the following fundamental result of Bogomolov (see [54, Proposition 2.2 ] for a nice and detailed proof).

Proposition A.2.1 Let $\mathcal{L} \subseteq \Omega_{X}^{1}$ be a line bundle, then $\mathcal{L}$ cannot be big.

This result has been generalized to log differential forms (see [14, Corollary 6.9]). 
Theorem A.2.2 (Bogomolov-Sommese Vanishing) Let $X$ be a smooth complex projective variety, $\mathcal{L}$ a line bundle, $C$ a normal crossing divisor on $X$. Then

$$
H^{0}\left(X, \Omega_{X}^{a}(\log C) \otimes \mathcal{L}^{-1}\right)=0
$$

for all $a<\kappa(X, \mathcal{L})$.

A fundamental geometric consequence of Bogomolov-Sommese vanishing is that sub-line bundles of the sheaf of log differentials cannot have many sections. More precisely, we have the following statement, which has already made an appearance as Corollary 3.3.8. We repeat the proof for the reader's convenience.

Corollary A.2.3 Let $X$ be a smooth projective surface, $C$ a normal crossing divisor on $X$. Then $\Omega_{X}^{1}(\log C)$ contains no big line bundles.

Proof. Note that $\mathcal{L}$ is big if and only if $\kappa(X, \mathcal{L})=2$. An inclusion $\mathcal{L} \hookrightarrow \Omega_{X}^{1}(\log C)$ gives rise to a non-trivial section of $H^{0}\left(X, \Omega_{X}^{1}(\log C) \otimes \mathcal{L}^{-1}\right)$, which vanishes according to Theorem A.2.2.

As a consequence we obtain numerical criteria for line bundles contained in $\Omega_{X}^{1}(\log C)$, with $C$ a normal crossing divisor on $X$.

Corollary A.2.4 Let $\mathcal{O}_{X}(D) \subseteq \Omega_{X}^{1}(\log C)$ be a line bundle, $\mathcal{O}_{X}(P)$ a nef line bundle on $X$. Then either $(P \cdot D) \leqslant 0$ or $\left(D^{2}\right) \leqslant 0$. In particular, if $D$ is effective, then $\left(D^{2}\right) \leqslant 0$.

Proof. Assuming $(P \cdot D)>0$ we will show $\left(D^{2}\right) \leqslant 0$. The linear system $\left|K_{X}-m D\right|=$ $\varnothing$ for large $m \geqslant 0$ because its intersection with the nef divisor $P$ becomes negative for large $m$ :

$$
\left(K_{X}-m D\right) \cdot P=K_{X} \cdot P-m P \cdot D<0 \text { for } m \gg 0 .
$$

By Serre duality,

$$
H^{2}\left(X, \mathcal{O}_{X}(m D)\right)=H^{0}\left(X, \mathcal{O}_{X}\left(K_{X}-m D\right)\right)=0,
$$

which implies via Riemann Roch that for all $m \gg 0$ we have

$$
\begin{aligned}
\gamma \cdot m & \geqslant h^{0}\left(X, \mathcal{O}_{X}(m D)\right) \geqslant h^{0}\left(X, \mathcal{O}_{X}(m D)\right)-h^{1}\left(X, \mathcal{O}_{X}(m D)\right) \\
& =\chi\left(X, \mathcal{O}_{X}(m D)\right)=\frac{\left(D^{2}\right)}{2} m^{2}+O(m) .
\end{aligned}
$$

The left-most inequality comes from the fact that $D$ is not big by Corollary A.2.3. It follows that $\left(D^{2}\right)$ must be non-positive.

Connecting up with the Hodge Index Theorem gives one of the main technical ingredients of the logarithmic Miyaoka-Yau inequality (see also [38, Proposition 1]). Note that the original statement requires $\operatorname{det} \mathcal{F}$ to be semi-ample but it is enough for the argument to assume $\operatorname{det} \mathcal{F}$ is nef. This proposition gives a nice criterion for vanishing of the group of global sections for certain vector bundles.

Proposition A.2.5 Let $\mathcal{F} \subseteq \Omega_{X}^{1}(\log C)$ be a locally free sheaf of rank 2 with nef determinant, $D$ an arbitrary divisor on $X$. If

$$
H^{0}\left(X, \mathcal{F} \otimes \mathcal{O}_{X}(-D)\right) \neq 0
$$

then

$$
(\operatorname{det} \mathcal{F} \cdot D) \leqslant \max \left\{0, c_{2}(\mathcal{F})\right\}
$$


Proof. Consider the projective bundle $\pi: V:=\mathbb{P}(\mathcal{F}) \rightarrow X$ associated to $\mathcal{F}$. Denoting by $H$ the tautological line bundle on $V$,

$$
H^{0}\left(V, \mathcal{O}_{V}\left(H-\pi^{*} D\right)\right) \simeq H^{0}\left(X, \mathcal{F} \otimes \mathcal{O}_{X}(-D)\right) \neq 0
$$

by assumption. Let $W \in\left|H-\pi^{*} D\right|$, and write it as

$$
W=W_{0}+\pi^{*} D^{\prime}
$$

where $W_{0} \sim H-\pi^{*}\left(D+D^{\prime}\right)$ is a prime divisor and $D^{\prime}$ a suitable effective (possibly trivial) divisor on $X$. The line bundle $\operatorname{det} \mathcal{F}$ is nef, therefore

$$
\left(\operatorname{det} \mathcal{F} \cdot D^{\prime}\right) \geqslant 0 \text {. }
$$

Setting $D^{\prime \prime}:=D+D^{\prime}$, we note that

$$
(\operatorname{det} \mathcal{F} \cdot D) \leqslant\left(\operatorname{det} \mathcal{F} \cdot D^{\prime \prime}\right),
$$

hence it suffices to prove the proposition for $D^{\prime \prime}$ in place of $D$. An application of the Hodge Index Theorem in the guise of Lemma A.2.6 gives that

$$
\left(\operatorname{det} \mathcal{F} \cdot D^{\prime \prime}\right) \leqslant c_{2}(\mathcal{F})+\left(D^{\prime \prime 2}\right) .
$$

The divisor $W_{0}$ gives rise to a non-trivial section $s \in H^{0}\left(X, \mathcal{F} \otimes \mathcal{O}_{X}\left(-D^{\prime \prime}\right)\right)$, which in turn embeds $\mathcal{O}_{X}\left(D^{\prime \prime}\right)$ into $\mathcal{F} \subseteq \Omega_{X}^{1}(\log C)$. Applying Corollary A.2.4 with the nef line bundle $\operatorname{det} \mathcal{F}$ results in

$$
\left(\operatorname{det} \mathcal{F} \cdot D^{\prime \prime}\right) \leqslant 0 \text { or }\left(D^{\prime \prime 2}\right) \leqslant 0,
$$

which, coupled with the inequality (5), finishes the proof.

Lemma A.2.6 With notation as above, let $W_{0} \in\left|H-\pi^{*} D^{\prime \prime}\right|$ be an irreducible divisor on $V$. Then

$$
(\operatorname{det} \mathcal{F} \cdot D) \leqslant c_{2}(\mathcal{F})+\left(D^{\prime \prime 2}\right) \text {. }
$$

Proof. This is [38, Lemma 8].

The following theorem, which is [38, Theorem 3], generalizes Proposition A.2.5 to symmetric powers. Its proof is based on the so called 'branched covering trick' [1. Theorem I.18.2] and Remark A.1.2.

Theorem A.2.7 Let $\mathcal{F} \subseteq \Omega_{X}^{1}(\log C)$ be a locally free sheaf of rank 2 with nef determinant bundle. If

$$
H^{0}\left(X, \operatorname{Sym}^{m} \mathcal{F} \otimes \mathcal{O}_{X}(-D)\right) \neq 0
$$

for some positive integer $m$, then

$$
(\operatorname{det} \mathcal{F} \cdot D) \leqslant \max \left\{0, m c_{2}(\mathcal{F})\right\} .
$$

Proof. Let $s \in H^{0}\left(X, \operatorname{Sym}^{m} \mathcal{F} \otimes \mathcal{O}_{X}(-D)\right)$ be a non-trivial section. By the aforementioned branched covering trick there exists a covering $f: Y \rightarrow X$ such that

$$
f^{*}(s) \in H^{0}\left(Y, \operatorname{Sym}^{m} f^{*} \mathcal{F} \otimes \mathcal{O}_{Y}\left(-f^{*} D\right)\right)
$$


can be written as

$$
f^{*}(s)=s_{1} \ldots s_{m}
$$

with $s_{i} \in H^{0}\left(Y, f^{*} \mathcal{F} \otimes \mathcal{O}_{Y}\left(-D_{i}\right)\right)$. Also note that $\left(\operatorname{det} f^{*} \mathcal{F}\right)^{\otimes m} \simeq f^{*}(\operatorname{det} \mathcal{F})^{\otimes m}$ is nef, and that we have canonical injections

$$
f^{*} \mathcal{F} \subseteq f^{*} \Omega_{X}^{1}(\log C) \subseteq \Omega_{Y}^{1}\left(\log f^{-1}(C)\right) .
$$

The second containment comes from [30, Proposition 11.2] because $f^{-1}(C)$ is a normal crossing divisor.

Proposition A.2.5 applied to the divisors $D_{i}$ then gives

$$
\left(\operatorname{det} f^{*} \mathcal{F} \cdot D_{i}\right) \leqslant \max \left\{c_{2}\left(f^{*} \mathcal{F}\right), 0\right\}
$$

for all $1 \leqslant i \leqslant m$. Summing them up and noting that $f^{*} D=\sum_{i} D_{i}$ we obtain

$$
\left(\operatorname{det} f^{*} \mathcal{F} \cdot f^{*} D\right)=\left(\operatorname{det} f^{*} \mathcal{F} \cdot \sum_{i=1}^{m} D_{i}\right) \leqslant \max \left\{m c_{2}\left(f^{*} \mathcal{F}\right), 0\right\} .
$$

If $d$ is the degree of the covering $f$, then

$$
\left(\operatorname{det} f^{*} \mathcal{F} \cdot f^{*} D\right)=d \cdot(\operatorname{det} \mathcal{F} \cdot D) \quad \text { and } \quad c_{2}\left(f^{*} \mathcal{F}\right)=d \cdot c_{2}(\mathcal{F}),
$$

which completes the proof.

Theorem A.2.8 (Logarithmic Miyaoka-Yau inequality) Let $X$ be a smooth complex projective surface, $C$ a semi-stable curve on $X$ such that $K_{X}+C$ is big. Then

$$
c_{1}\left(\Omega_{X}^{1}(\log C)\right)^{2} \leqslant 3 c_{2}\left(\Omega_{X}^{1}(\log C)\right),
$$

in other words,

$$
\left(K_{X}+C\right)^{2} \leqslant 3(e(X)-e(C)) .
$$

Remark A.2.9 We recall that $\operatorname{det} \Omega_{X}^{1}(\log C) \simeq \mathcal{O}_{X}\left(K_{X}+C\right)$ and $c_{2}\left(\Omega_{X}^{1}(\log C)\right)=$ $e(X-C)$, where $e$ denotes the topological Euler characteristic (see [44, Proposition $7.1])$.

Remark A.2.10 Let us quickly recall the definition of a semi-stable curve used here (following [4] ). A reduced 1-cycle $C \subseteq X$ is called semi-stable if

1. $C$ is a normal crossing divisor (i.e., has only ordinary nodes as singularities),

2. each smooth rational component of $C$ intersects the other components of $C$ in at least two points.

It is important to point out that an irreducible semi-stable curve can have ordinary nodes as singularities. If $C$ were defined to be simple normal crossing, instead of just normal crossing, then $C$ irreducible would imply $C$ smooth.

Remark A.2.11 With the notation of Theorem A.2.8, we will consider the following version of minimality (which could reasonably be called log-minimal). A smooth rational curve $E \subseteq X$ is called $C$-exceptional, if

$$
\left(E^{2}\right)=-1,(C \cdot E) \leqslant 1 .
$$

As outlined on [44, pp. 1-2], one can get rid of $C$-exceptional curves by blowing them down. 
Proof of Theorem A.2.8. According to Sakai's hint [44, Last sentence in the proof of Theorem 7.6] we will follow Miyaoka's original proof for $\Omega_{X}^{1}$.

Our plan is to apply Theorem A.2.7 to $\mathcal{F}=\Omega_{X}^{1}(\log C)$ itself. Exploiting Remark A.2.11, we may and will assume without loss of generality that $X$ contains no $C$-exceptional curves. As proven in [44, Theorem 5.8]2, $K_{X}+C$ is semi-ample provided it is big, hence $K_{X}+C$ is nef. Consequently, the determinant of $\Omega_{X}^{1}(\log C)$ is nef. We still have to fight for the existence of a non-trivial section of a suitable vector bundle.

If $c_{1}\left(\Omega_{X}^{1}(\log C)\right)^{2} \leqslant 2 c_{2}\left(\Omega_{X}^{1}(\log C)\right)$, then we are done. Hence we may assume

$$
c_{1}\left(\Omega_{X}^{1}(\log C)\right)^{2}>2 c_{2}\left(\Omega_{X}^{1}(\log C)\right)
$$

and set

$$
\alpha:=\frac{c_{2}\left(\Omega_{X}^{1}(\log C)\right)}{c_{1}\left(\Omega_{X}^{1}(\log C)\right)^{2}}<\frac{1}{2} .
$$

Here we used that $\alpha>0$, as shown in [44, Section 7]. Fix a sufficiently small number $\delta \in \mathbb{Q} \geqslant 0$. Observe that for sufficiently divisible $m>0$ we have

$$
\begin{aligned}
\left(\operatorname{det} \Omega_{X}^{1}(\log C) \cdot\left(m(\alpha+\delta)\left(K_{X}+C\right)\right)\right) & =m(\alpha+\delta)\left(\left(K_{X}+C\right)^{2}\right) \\
& =m\left((\alpha+\delta)\left(c_{1}\left(\Omega_{X}^{1}(\log C)\right)^{2}\right)\right. \\
& >m \cdot c_{2}\left(\Omega_{X}^{1}(\log C)\right) .
\end{aligned}
$$

By Theorem A.2.7, this means that

$$
H^{0}\left(X, \operatorname{Sym}^{m} \Omega_{X}^{1}(\log C) \otimes \mathcal{O}_{X}\left(-m(\alpha+\delta)\left(K_{X}+C\right)\right)\right)=0
$$

for all large $m$ such that $m(\alpha+\delta) \in \mathbb{Z}$. We next compute $H^{2}$ of the same vector bundle, with the help of Serre duality:

$$
\begin{array}{ll} 
& H^{2}\left(X, \operatorname{Sym}^{m} \Omega_{X}^{1}(\log C) \otimes \mathcal{O}_{X}\left(-m(\alpha+\delta)\left(K_{X}+C\right)\right)\right) \\
= & H^{0}\left(X, \mathcal{O}_{X}\left(K_{X}\right) \otimes\left(\operatorname{Sym}^{m} \Omega_{X}^{1}(\log C) \otimes \mathcal{O}_{X}\left(-m(\alpha+\delta)\left(K_{X}+C\right)\right)\right)^{*}\right) \\
= & \left.H^{0}\left(X, \operatorname{Sym}^{m} \Omega_{X}^{1}(\log C)\right)^{*} \otimes \mathcal{O}_{X}\left(K_{X}+m(\alpha+\delta)\left(K_{X}+C\right)\right)\right) \\
= & H^{0}\left(X,\left(\operatorname{Sym}^{m} \Omega_{X}^{1}(\log C) \otimes \mathcal{O}_{X}\left(-m\left(K_{X}+C\right)\right)\right) \otimes\right. \\
& \left.\mathcal{O}_{X}\left(K_{X}+m(\alpha+\delta)\left(K_{X}+C\right)\right)\right) \\
= & H^{0}\left(X, \operatorname{Sym}^{m} \Omega_{X}^{1}(\log C) \otimes \mathcal{O}_{X}\left(-m(1-\alpha-\delta)\left(K_{X}+C\right)+K_{X}\right)\right),
\end{array}
$$

where we used Remark A.1.2 for the third equality. Next we apply Theorem A.2.7 to the rank 2 vector bundle $\mathcal{F}=\Omega_{X}^{1}(\log C)$ and the divisor $D=m(1-\alpha-\delta)\left(K_{X}+\right.$ $C)-K_{X}$. As noted above, the determinant of $\mathcal{F}=\Omega_{X}^{1}(\log C)$ is nef and

$$
\begin{aligned}
(\operatorname{det} \mathcal{F} \cdot(m & \left.\left.(1-\alpha-\delta)\left(K_{X}+C\right)-K_{X}\right)\right) \\
& =\left(\left(K_{X}+C\right) \cdot\left(m(1-\alpha-\delta)\left(K_{X}+C\right)-K_{X}\right)\right) \\
& =m(1-\alpha-\delta)\left(\left(K_{X}+C\right)^{2}\right)-\left(\left(K_{X}+C\right) \cdot K_{X}\right) \\
& >m \cdot c_{2}(\mathcal{F})
\end{aligned}
$$

for all $m$ sufficiently large for which $m(1-\alpha-\delta) \in \mathbb{Z}$. Here we have used the observation that

$$
1-\alpha-\delta>0
$$

\footnotetext{
${ }^{2}$ Note that it is implicitly assumed in the proof of this result that $X$ is $\log$-minimal, this is why we had to get rid of the $C$-exceptional curves first.
} 
which follows from having $\alpha<\frac{1}{2}$ and having $\delta$ very small. In fact, we have

$$
1-\alpha-\delta \geqslant \alpha+\delta
$$

and therefore Theorem A.2.7 implies that

$$
H^{0}\left(X, \operatorname{Sym}^{m} \Omega_{X}^{1}(\log C) \otimes \mathcal{O}_{X}\left(-m(1-\alpha-\delta)\left(K_{X}+C\right)+K_{X}\right)\right)=0 .
$$

According to our computations using Serre duality, this is equivalent to

$$
H^{2}\left(X, \operatorname{Sym}^{m} \Omega_{X}^{1}(\log C) \otimes \mathcal{O}_{X}\left(-m(\alpha+\delta)\left(K_{X}+C\right)\right)\right)=0 .
$$

Consequently,

$$
\chi\left(X, \operatorname{Sym}^{m} \Omega_{X}^{1}(\log C) \otimes \mathcal{O}_{X}\left(-m(\alpha+\delta)\left(K_{X}+C\right)\right)\right) \leqslant 0 .
$$

On the other hand, the dictionary between the projective bundle

$$
\pi: V:=\mathbb{P}\left(\Omega_{X}^{1}(\log C)\right) \rightarrow X
$$

and $X$ (see [38], for example) gives

$$
\begin{array}{r}
\chi\left(X, \operatorname{Sym}^{m} \Omega_{X}^{1}(\log C) \otimes \mathcal{O}_{X}\left(-m(\alpha+\delta)\left(K_{X}+C\right)\right)\right) \\
=\chi\left(V, \mathcal{O}_{V}\left(m\left(H-(\alpha+\delta) \pi^{*}\left(K_{X}+C\right)\right)\right)\right),
\end{array}
$$

where the right hand side grows with $m$ asymptotically as

$$
\left.\frac{1}{6}\left(H-(\alpha+\delta) \pi^{*}\left(K_{X}+C\right)\right)\right)^{3} m^{3}
$$

by the asymptotic Riemann-Roch Theorem. As a result, we obtain

$$
\left.\left(H-(\alpha+\delta) \pi^{*}\left(K_{X}+C\right)\right)\right)^{3} \leqslant 0 .
$$

By letting $\delta \rightarrow 0$, we arrive at

$$
\left(H-\alpha \pi^{*}\left(K_{X}+C\right)\right)^{3} \leqslant 0 .
$$

To evaluate this, we recall the various intersection numbers on $V$ (see [38, Lemma $5])$ :

$\left(H^{3}\right)=c_{1}(\mathcal{F})^{2}-c_{2}(\mathcal{F}),\left(H^{2} \cdot \pi^{*} D\right)=(\operatorname{det} \mathcal{F} \cdot D),\left(H \cdot \pi^{*} D \cdot \pi^{*} D^{\prime}\right)=\left(D \cdot D^{\prime}\right)$.

Therefore,

$$
\begin{aligned}
\left(H-\alpha \pi^{*}\left(K_{X}+C\right)\right)^{3} & =\left(H^{3}\right)-3\left(H^{2} \cdot \alpha\left(\pi^{*}\left(K_{X}+C\right)\right)\right) \\
& +3\left(H \cdot\left(\alpha \pi^{*}\left(K_{X}+C\right)\right)^{2}\right)-\left(\left(\alpha \pi^{*}\left(K_{X}+C\right)\right)^{3}\right) \\
& =\left(c_{1}\left(\Omega_{X}^{1}(\log C)\right)^{2}-c_{2}\left(\Omega_{X}^{1}(\log C)\right)\right) \\
& -3 \alpha\left(c_{1}\left(\Omega_{X}^{1}(\log C)\right) \cdot\left(K_{X}+C\right)\right) \\
& +3 \alpha^{2}\left(\left(K_{X}+C\right)^{2}\right)+0 .
\end{aligned}
$$

Since $c_{1}\left(\Omega_{X}^{1}(\log C)\right)=\mathcal{O}_{X}\left(K_{X}+C\right)$ and $c_{2}\left(\Omega_{X}^{1}(\log C)\right)=\alpha c_{1}\left(\Omega_{X}^{1}(\log C)\right)^{2}$ (this latter by the definition of $\alpha$ ), after collecting terms we get

$$
\begin{aligned}
0 \geqslant\left(H-\alpha \pi^{*}\left(K_{X}+C\right)\right)^{3} & =\left(1-4 \alpha+3 \alpha^{2}\right) c_{1}\left(\Omega_{X}^{1}(\log C)\right)^{2} \\
& =(1-\alpha)(1-3 \alpha) c_{1}\left(\Omega_{X}^{1}(\log C)\right)^{2} .
\end{aligned}
$$

As $0 \leqslant \alpha<\frac{1}{2}$ and $c_{1}\left(\Omega_{X}^{1}(\log C)\right)>0$ (since $K_{X}+C$ is big and nef), we conclude that $3 \alpha \geqslant 1$, which is what we wanted to show. 
Acknowledgement. We would like to thank the Mathematisches Forschungsinstitut Oberwolfach for providing a perfect venue for our workshop. We also thank Lawrence Ein, Laurent Evain, Jun-Muk Hwang and Roberto Muñoz, whose contributions to these notes through their active participation during the workshop we gratefully acknowledge, and Nick Shepherd-Barron for his comments regarding the bounded negativity problem. Finally, we are grateful to Stefan Kebekus for suggesting the use of subversion for helping to make possible the preparation of an article with so many coauthors and for his mathematical comments and support for the project.

\section{References}

[1] W. P. Barth, K. Hulek, C. A. M. Peters, A. Van de Ven. Compact complex surfaces. Second edition. Ergebnisse der Mathematik und ihrer Grenzgebiete. 3. Folge. 4. Springer-Verlag, Berlin, 2004.

[2] Thomas Bauer, Sandra Di Rocco, Brian Harbourne, Michał Kapustka, Andreas Knutsen, Wioletta Syzdek, and Tomasz Szemberg. A primer on Seshadri constants, Interactions of classical and numerical algebraic geometry, Contemp. Math., vol. 496, Amer. Math. Soc., Providence, RI, 2009, pp. 33-70.

[3] C. Bocci, L. Chiantini. The effect of points fattening on postulation, Journal of Pure and Applied Algebra 215 (2011), 89-98.

[4] C. Bocci, B. Harbourne. Comparing powers and symbolic powers of ideals, J. Algebraic Geometry, 19 (2010), 399-417.

[5] F. Bogomolov. Holomorphic tensors and vector bundles on projective varieties, Math. USSR Isvestija 13 (1979), 499-555.

[6] J. Bourgain, N. Katz, T. Tao. A sum-product estimate in finite fields, and applications, Geom. Funct. Anal. 14 (2004), 27-57.

[7] C. Ciliberto, R. Miranda. Linear systems of plane curves with base points of equal multiplicity, Trans. Amer. Math. Soc. 352, 40374050 (2000).

[8] G. V. Chudnovsky. Singular points on complex hypersurfaces and multidimensional Schwarz Lemma, Séminaire de Théorie des Nombres, Paris 1979-80, Séminaire Delange-Pisot-Poitou, Progress in Math vol. 12, M-J Bertin, editor, Birkhäuser, Boston-Basel-Stuttgart (1981).

[9] D. Cohen, A. Suciu. Characteristic varieties of arrangements, Math. Proc. Cambridge Phil. Soc. 127 (1999), 33-53.

[10] J.-P. Demailly. Singular Hermitian metrics on positive line bundles. Complex algebraic varieties (Bayreuth, 1990), Lect. Notes Math. 1507, Springer-Verlag, 1992, pp. 87-104.

[11] Jean-Pierre Demailly, Lawrence Ein, and Robert Lazarsfeld. A subadditivity property of multiplier ideals, Michigan Math. J. 48 (2000), 137-156, Dedicated to William Fulton on the occasion of his 60th birthday.

[12] M. Dumnicki. Reduction method for linear systems of plane curves with base points, Ann. Polon. Math. 90 (2007), 131-143.

[13] Lawrence Ein, Robert Lazarsfeld, and Karen E. Smith. Uniform bounds and symbolic powers on smooth varieties, Invent. Math. 144 (2001), no. 2, 241-252.

[14] H. Esnault, E. Viehweg. Lectures on vanishing theorems, DMV Seminar, 20. Birkhäuser Verlag, Basel, 1992.

[15] L. Évain. Computing limit linear series with infinitesimal methods, Annales de l'institut Fourier, 57 (2007), p. 1947-1974.

[16] M. Falk, S. Yuzvinsky. Multinets, resonance varieties, and pencils of plane curves, Compositio Math. 143 (2007), 1069-1088.

[17] W. Fulton. Intersection theory. Ergeb. Math. Grenzgeb. (3) 2, Springer-Verlag, 1984.

[18] W. Fulton. Introduction to toric varieties. Annals of Mathematics Studies (1993), Princeton University Press.

[19] A. V. Geramita, A. Gimigliano, B. Harbourne. Projectively Normal but Superabundant Embeddings of Rational Surfaces in Projective Space, J. Alg. 169 (1994), 791-804.

[20] A. Gimigliano. On Linear Systems of Plane Curves, Ph. D. thesis, Queen's University, Kingston, Ontario (1987).

[21] B. Harbourne. Global aspects of the geometry of surfaces, Ann. Univ. Pedagog. Crac. Stud. Math. 11 (2010), 5-41. 
[22] B. Harbourne. Anticanonical rational surfaces, Trans. Amer. Math. Soc. 349, 1191-1208 (1997).

[23] B. Harbourne. The geometry of rational surfaces and Hilbert functions of points in the plane, Canadian Mathematical Society Conference Proceedings 6 , 95-111 (1986).

[24] B. Harbourne, J. Roé. Extendible Estimates of multi-point Seshadri Constants, preprint 2003, arXiv math/0309064v1.

[25] R. Hartshorne. Algebraic Geometry, Graduate texts in mathematics (52), New York, SpringerVerlag, 496 pp., 1977.

[26] A. Hirschowitz. La méthode d'Horace pour l'interpolation à plusieurs variables, Manuscr. Math. 50 (1985), 337-388.

[27] A. Hirschowitz. Une conjecture pour la cohomologie des diviseurs sur les surfaces rationelles génériques, Jour. Reine Angew. Math. 397 (1989), 208-213.

[28] M. Hochster, C. Huneke. Comparison of symbolic and ordinary powers of ideals, Invent. Math. 147 (2002), no. 2, 349-369.

[29] D. Huybrechts, M. Lehn. The geometry of moduli spaces of sheaves. Aspects of Mathematics, E31. Vieweg 1997.

[30] S. Iitaka. Algebraic geometry: an introduction to birational geometry of algebraic varieties. Graduate Texts in Mathematics 76., Springer-Verlag, 1982.

[31] J. Kollár. Nonrational hypersurfaces, J. Amer. Math. Soc. 8 (1995), 241-249.

[32] J. Kollár. Shafarevich maps and plurigenera of algebraic varieties, Invent. Math. 113 (1993) 177-215.

[33] A. Küronya. Asymptotic cohomological functions on projective varieties. Amer. J. Math. 128 (2006), 1475-1519.

[34] H. Lange, C. Birkenhake. Complex Abelian Varieties. Grundlehren der math. Wiss. 302, Springer-Verlag, 1992.

[35] R. Lazarsfeld. Positivity in Algebraic Geometry I.-II. Ergebnisse der Mathematik und ihrer Grenzgebiete, Vols. 48-49., Springer Verlag, Berlin, 2004.

[36] A. Libgober, S. Yuzvinsky. Cohomology of the Orlik-Solomon algebras and local systems, Compositio Math. 121 (2000), 337-361.

[37] R.A. Lorentz. Multivariate Hermite interpolation by algebraic polynomials: A survey, J. Comp. Appl. Math. 112 (2000), 167-201.

[38] Y. Miyaoka. On the Chern numbers of surfaces of general type, Inv. Math. 42 (1977), 225-237.

[39] Mircea Mustaţă, On multiplicities of graded sequences of ideals, J. Algebra 256 (2002), no. 1, $229-249$.

[40] M. Nagata. On the 14-th problem of Hilbert, Amer. J. Math. 81 (1959), 766-772.

[41] P. Orlik, L. Solomon. Combinatorics and topology of complements of hyperplanes, Invent. Math. 56 (1980), 167-189.

[42] P. Orlik, H. Terao. Commutative algebras for arrangements, Nagoya Math Journal, 134 (1994), 65-73.

[43] J. Roé. Maximal rank for planar singularities of multiplicity 2, J. Alg. 302 (2006), 37-54.

[44] F. Sakai. Semi-stable curves on algebraic curves and logarithmic pluricanonical maps, Math. Ann. 254 (1980), 89-120.

[45] H. Schenck. Resonance varieties via blowups of $\mathbb{P}^{2}$ and scrolls, IMRN 10.1093/imrn/rnq271 (2010).

[46] B. Segre. Alcune questioni su insiemi finiti di punti in Geometria Algebrica, Atti del Convegno Internaz. di Geom. Alg., Torino (1961), 15-33.

[47] H. Skoda. Estimations $L^{2}$ pour l'opérateur $\widehat{\partial}$ et applications arithmétiques, in: Séminaire P. Lelong (Analyse), 1975/76, Lecture Notes in Mathematics 578, Springer, 1977, 314-323.

[48] B. Sturmfels. Four counterexamples in combinatorial algebraic geometry, J. Algebra 230 (2000), 282-294.

[49] A. Suciu, Fundamental groups of line arrangements: Enumerative aspects, Contemporary Math. 276 (2001), AMS, Providence, RI, 43-79.

[50] Irena Swanson, Linear equivalence of ideal topologies, Math. Z. 234 (2000), no. 4, 755-775.

[51] E. Szemerédi, and W. T. Trotter. Extremal problems in discrete geometry, Combinatorica 3 (1983), 381-392.

[52] Shunsuke Takagi and Ken-ichi Yoshida. Generalized test ideals and symbolic powers, Michigan Math. J. 57 (2008), 711-724.

[53] Zach Teitler, Bounding symbolic powers via asymptotic multiplier ideals, Ann. Univ. Paedagog. Crac. Stud. Math. 8 (2009), 67-77.

[54] A. Van de Ven. Some recent results on surfaces of general type, Séminaire Bourbaki, 29e année (1976/77), Exp. No. 500, pp. 155-166, Lecture Notes in Math., 677, Springer 1978.

[55] L. Anh Vinh. Szemeredi-Trotter type theorem and sum-product estimate in finite fields, Euro- 
pean Journal of Combinatorics, to appear (arXiv:0711.4427).

[56] M. Waldschmidt. Propriétés arithmétiques de fonctions de plusieurs variables II, Séminaire P. Lelong (Analyse), 1975-76, Lecture Notes Math. 578, Springer-Verlag, 1977, 108-135.

[57] G. Ziegler. Lectures on polytopes. Graduate Texts in Mathematics 152 (1995), Springer.

Thomas Bauer, Fachbereich Mathematik und Informatik, Philipps-Universität Marburg, Hans-Meerwein-Straße, D-35032 Marburg, Germany.

E-mail address: tbauer@mathematik.uni-marburg.de

Cristiano Bocci, Dipartimento di Scienze Matematiche e Informatiche "R. Magari", Università di Siena, Pian dei Mantellini, 4453100 Siena, Italy.

E-mail address: bocci24@unisi.it

Susan Cooper, Department of Mathematics, University of Nebraska-Lincoln, Lincoln, NE 68588-0130, USA.

E-mail address: scooper4@math.unl.edu

Sandra Di Rocco, Department of Mathematics, Royal Institute of Technology (KTH), S-10044 Stockholm, Sweden.

E-mail address: dirocco@math.kth.se

Marcin Dumnicki, Institute of Mathematics, Jagiellonian University ul. Łojasiewicza 6, 30-348 Kraków, Poland.

E-mail address: Marcin.Dumnicki@im.uj.edu.pl

Brian Harbourne, Department of Mathematics, University of Nebraska-Lincoln, Lincoln, NE 68588-0130, USA.

E-mail address: bharbour@math.unl.edu

Kelly Jabbusch, Department of Mathematics, Royal Institute of Technology (KTH), S-10044 Stockholm, Sweden.

E-mail address: jabbusch@math.kth.se

Andreas Leopold Knutsen, Department of Mathematics, University of Bergen, Johs. Brunsgt. 12, N-5008 Bergen, Norway.

E-mail address: andreas.knutsen@math.uib.no

Alex Küronya, Budapest University of Technology and Economics, Mathematical Institute, Department of Algebra, Pf. 91, H-1521 Budapest, Hungary.

E-mail address: alex.kuronya@math.bme.hu

Current address: Alex Küronya, Albert-Ludwigs-Universität Freiburg, Mathematisches Institut, Eckerstraße 1, D-79104 Freiburg, Germany.

Rick Miranda, Department of Mathematics, Colorado State University, Fort Collins, CO 80523, USA.

E-mail address: rick.miranda@colostate.edu

Joaquim Roé, Departament de Matemàtiques, Universitat Autònoma de Barcelona E08193 Bellaterra (Barcelona), Spain.

E-mail address: jroe@mat.uab.cat

Hal Schenck, Mathematics Department, Univ. Illinois, Urbana, Illinois, 61801 USA.

E-mail address: schenck@math.uiuc.edu

Tomasz Szemberg, Instytut Matematyki UP, PL-30-084 Kraków, Poland.

E-mail address: tomasz.szemberg@uni-due.de 
Current address: Tomasz Szemberg, Albert-Ludwigs-Universität Freiburg, Mathematisches Institut, Eckerstraße 1, D-79104 Freiburg, Germany.

Zach Teitler, Department of Mathematics, Boise State University, 1910 University Drive, Boise, ID 83725-1555, USA.

E-mail address: zteitler@boisestate.edu 